\title{
A SECOND LOOK OF SOBOLEV SPACES ON METRIZABLE GROUPS
}

\author{
Przemysław Górka and Tomasz Kostrzewa \\ Warsaw University of Technology, Department of Mathematics and Information Sciences \\ Ul. Koszykowa 75, 00-662 Warsaw, Poland; pgorka@mini.pw.edu.pl \\ Warsaw University of Technology, Department of Mathematics and Information Sciences \\ Ul. Koszykowa 75, 00-662 Warsaw, Poland; kostrzewat@mini.pw.edu.pl
}

\begin{abstract}
We continue our study of Sobolev spaces on locally compact abelian groups. In this paper we mainly focus on the case of metrizable groups. We show the density of the Bruhat-Schwartz space in Sobolev space. We prove the trace theorem on the cartesian product of topological groups. The comparison of Sobolev and fractional Sobolev spaces are given. In particular, it is proved that in the case of any abelian connected Lie group Sobolev and fractional Sobolev spaces coincide. Most of the theorems are illustrated by $p$-adic groups.
\end{abstract}

\section{Introduction}

Sobolev spaces are the main tools in the modern theory of partial differential equations. They give a very natural functional analytical framework for the study of existence, regularity and qualitative properties of the boundary value problems. Such kind of spaces are well understood on the Euclidean space $\mathbf{R}^{n}$ and sufficiently regular subsets of $\mathbf{R}^{n}$ (see e.g. [2, 42]), complete Riemannian manifolds [5, 23]. On the other hand, Hajłasz [21] (see also [22]) and Shanmugalingam [36] introduced the Sobolev spaces on metric measure spaces. Such kind of spaces have found applications in differential equations e.g. harmonic functions on metric measure spaces [37]. There are also some works on Sobolev spaces in the $p$-adic context (see $[28,33]$ and references therein), and in special cases of locally compact groups such as the Heisenberg group (see $[6,10])$.

The purpose of this paper is to continue the investigation of Sobolev spaces on metrizable locally compact abelian (LCA) groups. The Sobolev spaces on LCA groups have been introduced by Górka and Reyes in [20], where Sobolev embedding and Rellich-Kondrachov theorem have been proved (see also [16]). In [19] we improved theorems about continuous and compact embeddings (see also [18] for related result in the case of countable dual groups). Moreover, in [19] we introduced Sobolev spaces on subsets of LCA groups and we proved analogue of Rellich lemma for those spaces. In [17] we have studied Sobolev spaces on locally compact abelian groups in the case when the dual group is metrizable. We hope that the theory developed here will shed a new light on the theory of differential equations on metric measure spaces, e.g. harmonic functions $[1,12]$.

The remainder of the paper is organized as follows. In Preliminaries we review some definitions from harmonic analysis and the theory of Sobolev spaces on topological groups. Moreover, some general remarks about metrizable groups are given

https://doi.org/10.5186/aasfm.2020.4507

2010 Mathematics Subject Classification: Primary 32A99, 46E35, $22 B 99$.

Key words: Abstract harmonic analysis, Sobolev spaces, analysis on metric measure spaces, trace theorem, fractional Sobolev spaces, metric group. 
and the Bruhat-Schwartz spaces are introduced. Then, in Section 3 assuming that the Bruhat-Schwartz space is contained in the Sobolev space we are able to prove the density of the Bruhat-Schwartz in the Sobolev space. Moreover, we provide the class of admissible weights, i.e. such that the Bruhat-Schwartz space is contained in the Sobolev space. Section 4 is devoted to studying the properties of the BruhatSchwartz space on the cartesian product of groups. Such analysis is crucial to prove the trace theorem in Section 5. In that section we assume that the dual group is metrizable and the dual measure is upper $\beta$-regular (see Preliminaries for definitions). Furthermore, in Section 5 we prove that in the case of $p$-adic numbers the trace operator is surjective. In Section 6 we compare our space with the fractional Sobolev space. Assuming that the group admits invariant metric and the Haar measure is $\beta$-regular, we obtain some general result about the continuous embedding of the Sobolev space in the fractional Sobolev space. We show that in the case of connected abelian Lie group, Sobolev space and fractional Sobolev space coincide. The paper is closed with the Appendix, where some basic facts about $p$-adic numbers are contained.

\section{Preliminaries}

2.1. Sobolev spaces. We use standard notations from harmonic analysis (see [11] and [24]). Let us fix a locally compact abelian group $G$. We denote by $\mu_{G}$ the unique Haar measure of $G$ and by $G^{\wedge}$ the dual group of the group $G$ that is, $G^{\wedge}$ is the locally compact abelian group of all continuous group homomorphisms from $G$ to the circle group $\mathbf{T}$. We denote by $\hat{\mu}_{G}$ Haar measure (Plancherel measure) on $G^{\wedge}$. By $e$ (resp. $\hat{e})$ we shall denote the identity of $G$ (resp. $\hat{G}$ ). The $L^{p}$ spaces over $G$ are defined as usual,

$$
L^{p}(G)=\left\{f: G \rightarrow \mathbf{C}: \int_{G}|f(x)|^{p} d \mu_{G}(x)<\infty\right\},
$$

and we set

$$
\|f\|_{L^{p}(G)}=\left(\int_{G}|f(x)|^{p} d \mu_{G}(x)\right)^{1 / p} .
$$

Since the definition of the Sobolev space on locally compact abelian group uses essentially the Fourier transform, we also recall that the Fourier transform on $G$ is defined as follows: if $f \in L^{1}(G)$, then it Fourier transform is the function $\hat{f}: G^{\wedge} \rightarrow \mathbf{C}$ given by

$$
\hat{f}(\xi)=\int_{G} \overline{\xi(x)} f(x) d \mu_{G}(x) .
$$

Now, let us recall the notion of the Sobolev spaces on locally compact abelian groups (see [20], [18] and [19]).

Definition 2.1. Let us fix a map $\gamma: G^{\wedge} \rightarrow[0, \infty)$ and a nonnegative real number $s$. We shall say that $f \in L^{2}(G)$ belongs to the Sobolev space $H_{\gamma}^{s}(G)$ if the following integral is finite:

$$
\int_{G^{\wedge}}\left(1+\gamma(\xi)^{2}\right)^{s}|\hat{f}(\xi)|^{2} d \hat{\mu}_{G}(\xi) .
$$

Moreover, for $f \in H_{\gamma}^{s}(G)$ its norm $\|f\|_{H_{\gamma}^{s}(G)}$ is defined as follows:

$$
\|f\|_{H_{\gamma}^{s}(G)}=\left(\int_{G^{\wedge}}\left(1+\gamma(\xi)^{2}\right)^{s}|\hat{f}(\xi)|^{2} d \hat{\mu}_{G}(\xi)\right)^{1 / 2} .
$$


For the basic properties of this kind of spaces we refer to [20].

2.2. Metric groups. We now briefly recall the theory of metrizable locally compact groups. Let us recall that the Birkhoff-Kakutani theorem [25] states that the LCA group is metrizable if and only if it is first countable. On the other hand, by the Struble theorem [39], we know that on a topological group exists proper ${ }^{1}$ and invariant (i.e. $d(x, y)=d(g x, g y)$ for all $x, y, g \in G)$ metric if and only if the group is second countable. Furthermore, if $G$ is LCA and second countable, then the dual group $G^{\wedge}$ is second countable too (see [8]).

Next, we recall some definitions.

Definition 2.2. [17] Let $G$ be an LCA group. We say that metrics $d: G \times G \rightarrow$ $\mathbf{R}_{+}$and $\hat{d}: G^{\wedge} \times G^{\wedge} \rightarrow \mathbf{R}_{+}$are dual metrics if for each character $\xi \in G^{\wedge}$ and every $x, y \in G$ we have

$$
|\xi(x)-\xi(y)| \leq \hat{d}(\xi, \hat{e}) d(x, y) .
$$

One can easily convince oneself that $\mathbf{R}^{n}, \mathbf{T}^{n}$ and $\mathbf{Q}_{p}^{n}$ with natural metrics satisfy condition of the above definition ${ }^{2}$.

Definition 2.3. [17] Let $(G, d, \mu)$ be an LCA group with metric $d$ and the Haar measure $\mu$. We shall say that the measure $\mu$ is upper $\beta$-regular if:

- $G$ is not discrete and there exists a constant $D_{\beta}>0$, such that for all $r>0$ we have

$$
\mu(B(e, r)) \leq D_{\beta} r^{\beta}
$$

- or, $G$ is discrete and there exists $R_{0}>0$ and $D_{\beta}>0$ such that $B\left(e, R_{0}\right)=\{e\}$ and for $r \geq R_{0}$

$$
\mu(B(e, r)) \leq D_{\beta} r^{\beta}
$$

Remark 2.1. The number $\beta$ in Definition 2.3 can be treated as an analogue of the dimension for groups. Very similar condition appears in the analysis on metric measure spaces (see $[26,22])$.

Remark 2.2. Assuming $\beta$-regularity of the dual measure $\hat{\mu}$, the continuity of the embeddings of the Sobolev space $H_{\gamma}^{s}$ have been proved [17], i.e. Sobolev inequality (Theorem 1 in [17]), Morrey theorem (Theorem 2 in [17]) and the Moser-Trudinger inequality (Theorem 3 in [17]).

Example 1. Suppose $G$ and $H$ are LCA groups with metrics $d$ and $\rho$ respectively. If $\mu_{G}$ is upper $n$-regular and $\mu_{H}$ is upper $k$-regular then the Haar measure $\mu_{G \times H}$ on $G \times H$ is $(n+k)$-regular with respect to the metric

$$
(d \times \rho)\left(\left(g_{1}, h_{1}\right),\left(g_{2}, h_{2}\right)\right)=\max \left\{d\left(g_{1}, g_{2}\right), \rho\left(h_{1}, h_{2}\right)\right\} .^{3}
$$

Having in mind the above example, we can produce the next ones.

Example 2. The Haar measure on $\mathbf{Q}_{p}^{n}$ is upper $n$-regular.

Example 3. The Haar measure on $\mathbf{Z}^{d}$ is upper $d$-regular.

In the sequel we shall need the following result.

\footnotetext{
${ }^{1}$ On a topological space $X$, metric $d$ is called proper if balls are relatively compact.

${ }^{2}$ Dual groups of $\mathbf{R}^{n}, \mathbf{T}^{n}$ and $\mathbf{Q}_{p}^{n}$ are $\mathbf{R}^{n}, \mathbf{Z}^{n}$ and $\mathbf{Q}_{p}^{n}$ respectively.

${ }^{3}$ It easily follows from the fact that balls with respect to metric $d \times \rho$ are cartesian products of balls with respect to metrics $d$ and $\rho$ and the Haar measure on $G \times H$ is the product measure of $\mu_{G}$ and $\mu_{H}$.
} 
Lemma 2.1. Let $G$ be an LCA group which is metrizable with metric d. Suppose that the Haar measure $\mu_{G}$ on $G$ is upper $\beta$-regular. If $s>\frac{\beta}{2}$, then there exists $C$ such that for every $R>0$ the following inequality

$$
\int_{G \backslash B(e, R)} \frac{d \mu_{G}(x)}{\left(1+d(e, x)^{2}\right)^{s}} \leq C R^{\beta-2 s}
$$

holds.

Proof. The lemma is very similar to Lemma 1 in [17]. Nevertheless, for the convenience of the reader we shall give the proof. The proof falls naturally into two steps.

(1) $G$ is not discrete. Since $\beta-2 s<0$, we have

$$
\begin{aligned}
\int_{G \backslash B(e, R)} \frac{d \mu_{G}(x)}{\left(1+d(x, e)^{2}\right)^{s}} & =\sum_{k=0}^{\infty} \int_{B\left(e, 2^{k+1} R\right) \backslash B\left(e, 2^{k} R\right)} \frac{d \mu_{G}(x)}{\left(1+d(x, e)^{2}\right)^{s}} \\
& \leq D_{\beta} \sum_{k=0}^{\infty} \frac{\left(2^{k+1} R\right)^{\beta}}{2^{2 s k} R^{2 s}}=\frac{2^{\beta} D_{\beta} R^{\beta-2 s}}{1-2^{\beta-2 s}} .
\end{aligned}
$$

(2) $G$ is discrete. If $R \geq R_{0}$, then the proof runs as before. Now, for $R<R_{0}$ we have

$$
\int_{G \backslash B(e, R)} \frac{d \mu_{G}(x)}{\left(1+d(x, e)^{2}\right)^{s}}=\int_{G \backslash B\left(e, R_{0}\right)} \frac{d \mu_{G}(x)}{\left(1+d(x, e)^{2}\right)^{s}} \leq D_{\beta} \frac{2^{\beta} R_{0}^{\beta-2 s}}{1-2^{\beta-2 s}} \leq D_{\beta} \frac{2^{\beta} R^{\beta-2 s}}{1-2^{\beta-2 s}} .
$$

2.3. Bruhat-Schwartz classes. Bruhat-Schwartz class was first introduced by Bruhat in [9]. We will use one of the equivalent definition given by Osborne in [32]. It turns out that this class is very suitable for the theory of Sobolev spaces on LCA groups.

For the convenience of the reader we recall the relevant material from [32] without proofs, thus making our exposition self-contained.

Assume that $S$ is a symmetric subset of an abelian group $G$, i.e. $S=S^{-1}$. Denote by $\langle S\rangle$ the smallest subgroup of $G$ containing $S$ i.e.

$$
\langle S\rangle=\left\{a_{1} a_{2} \ldots a_{n} b_{1}^{-1} b_{2}^{-1} \ldots b_{m}^{-1}: a_{i}, b_{j} \in S, n, m \geq 1\right\},
$$

where $S^{n}=\left\{a_{1} \cdots a_{n}: a_{1}, \ldots, a_{n} \in S\right\}$.

Remark 2.3. From symmetry of $S$ it follows that

$$
\langle S\rangle=\left\{a_{1} a_{2} \ldots a_{n}: a_{i} \in S, n \geq 1\right\}=\bigcup_{n \geq 1} S^{n} .
$$

Definition 2.4. [32] Let $G$ be a locally compact group. We say that a function $f \in L^{\infty}(G)$ belongs to the class $\mathcal{A}(G)$ if there exists a compact set $C(f) \subset G$ such that for all $n \geq 1$ there is a positive constant $M_{n}$ such that for all $k \geq 1$

$$
\left\|\left.f\right|_{G \backslash C(f)^{k}}\right\|_{L^{\infty}(G)} \leq M_{n} k^{-n}
$$

Remark 2.4. [32]

(i) Sets $C(f)$ can be enlarged at will i.e. we can add to it any compact set without changing the inequality in the definition of $\mathcal{A}(G)$. From now on we will assume that $C(f)$ contains an open neighbourhood of the identity of $G$.

(ii) $\mathcal{A}(G) L^{\infty}(G) \subset \mathcal{A}(G)$. 
(iii) $f$ vanishes $\mu$-a.e., off $\langle C(f)\rangle$.

In general, when some function $f \in \mathcal{A}(G)$ is given, it might be very hard to find a compact set $C(f)$ from the above definition. This gets even more complicated if we need to find those sets for more then one function. Next lemma gives us a simple solution for such problems.

Lemma 2.2. Let $G$ be an LCA group which is compactly generated i.e. there exists a compact set such that

$$
G=\bigcup_{n=1}^{\infty}\left(K \cup K^{-1}\right)^{n} .
$$

Then there exists a compact set $C$ which is an admissible set for any $f \in \mathcal{A}(G)$ i.e. for any $n$ there exists $M_{n}$ such that for every $k$

$$
\left\|\left.f\right|_{G \backslash C^{k}}\right\|_{L^{\infty}(G)} \leq M_{n} k^{-n}
$$

Proof. Let us take $f \in \mathcal{A}(G)$. Then there exists a compact set $C(f)$ such that for all $n$ there exists $M_{n}(f)$ such that for all $j$ the inequality holds

$$
|f(x)| \leq M_{n}(f) j^{-n}
$$

for almost all $x \in G \backslash C(f)^{j}$. Since $G$ is compactly generated, there exists an open neighbourhood $U$ of $e_{G}$ with compact closure $C:=\bar{U}$ such that

$$
G=\bigcup U^{n}=\bigcup C^{n} \text {. }
$$

From this, it follows that there exists $m(f)$ such that $C(f) \subset C^{m(f)}$. Thus, without loss of generality we can assume that $C(f)=C^{m(f)}$. Observing that

$$
M_{n}(f) j^{-n}=M_{n}(f)(m(f))^{n}(m(f) j)^{-n}
$$

and denoting $M_{n}(f)^{\prime}=M_{n}(f) m(f)^{n}$, we get that

$$
|f(x)| \leq M_{n}(f)^{\prime}(m(f) j)^{-n}
$$

for almost all $x \in G \backslash C(f)^{j}=G \backslash C^{m(f) j}$. To prove that $C$ can be taken as a compact set in the definition of $f \in \mathcal{A}(G)$ we need to show the above inequality for all natural numbers. In order to do that, we only need to show an appropriate inequality with $k=m(f) j+1, m(f) j+2, \ldots, m(f) j+m(f)-1$. To do that fix $k=m(f) j+s$ for some $s \in\{1,2, \ldots, m(f)-1\}$ and observe that

$$
M_{n}^{\prime}(f)(m(f) j)^{-n} \leq M_{n}(f)^{\prime} 2^{n}(m(f) j+s)^{-n} .
$$

Denote $M_{n}^{\prime \prime}(f)=2^{n} M_{n}^{\prime}(f)$. Since $G \backslash C^{m(f) j+s} \subset G \backslash C^{m(f) j}$, we can apply inequality (1) to get for almost all $x \in G \backslash C^{m(f) j+s}$ the inequality

$$
|f(x)| \leq M_{n}^{\prime}(f)(m(f) j)^{-n} \leq M_{n}^{\prime \prime}(f)(m(f) j+s)^{-n} .
$$

Therefore, we have proved that $C$ is an admissible set for any function $f \in \mathcal{A}(G)$.

From the proof of the above lemma, we get the following corollary.

Corollary 2.1. Let $G$ be an LCA group which is compactly generated. Suppose that $U$ is a subset of $G$ satisfying

(i) $U$ is an open neighbourhood of the identity,

(ii) $\bar{U}$ is compact,

(iii) $G=\bigcup U^{n}$.

Then $C:=\bar{U}$ is an admissible set for all $f \in \mathcal{A}(G)$. 
Lemma 2.3. [32] Let $G$ be a locally compact group. If $f \in \mathcal{A}(G)$ then for each positive integer $n$ there exists a constant $M_{n}^{\prime}$ such that for all $k \geq 1$ we have

$$
\int_{C(f)^{k+1} \backslash C(f)^{k}}|f(x)| d \mu(x) \leq M_{n}^{\prime} k^{-n} .
$$

Corollary 2.2. [32] Let $G$ be a locally compact abelian group. Then $\mathcal{A}(G) \subset$ $L^{1}(G)$, and for all $n$ there exists a constant $M_{n}^{\prime \prime}$ such that for all $k \geq 1$

$$
\left\|\left.f\right|_{G \backslash C(f)^{k}}\right\|_{L^{1}(G)} \leq M_{n}^{\prime \prime} k^{-n} \text {. }
$$

Next, we introduce the Bruhat-Schwartz class $\mathcal{S}$.

Definition 2.5. [32] Let $G$ be a locally compact abelian group. We say that $f$ belongs to the Bruhat-Schwartz class $\mathcal{S}(G)$ if $f \in \mathcal{A}(G)$ and $\hat{f} \in \mathcal{A}\left(G^{\wedge}\right)$.

Remark 2.5. From Corollary 2.2 we have that $\mathcal{A}(G) \subset L^{1}(G)$. Since $\mathcal{A}(G) \subset$ $L^{\infty}(G)$ it follows that $\mathcal{A}(G) \subset L^{p}(G)$ for any $p \geq 1$. Indeed, from the Hölder inequality we have

$$
\int_{G}|f(x)|^{p} d \mu(x) \leq\|f\|_{L^{\infty}(G)}^{p-1}\|f\|_{L^{1}(G)} .
$$

It follows that if $f \in \mathcal{S}(G)$, then $f \in L^{p}(G)$ and $\hat{f} \in L^{p}\left(G^{\wedge}\right)$ for any $p \geq 1$.

Indirect application of Corollary 2.1 leads us to the following examples.

Example. ${ }^{4}$

- $\mathcal{S}\left(\mathbf{R}^{n}\right)=\left\{f \in C^{\infty}\left(\mathbf{R}^{n}\right): \forall \alpha, \beta \sup _{x \in \mathbf{R}^{n}}\left|x^{\alpha} \partial^{\beta} f(x)\right|<\infty\right\}$,

- $\mathcal{S}\left(\mathbf{Z}^{l}\right)=\left\{f: \mathbf{Z}^{l} \rightarrow \mathbf{C}: \forall M>0 \sup _{x \in \mathbf{Z}^{l}}\left(1+\|x\|^{2}\right)^{M}|f(x)|<\infty\right\}$,

- $\mathcal{S}\left(\mathbf{T}^{n}\right)=C^{\infty}\left(\mathbf{T}^{n}\right)$,

- $\mathcal{S}\left(\mathbf{Q}_{p}^{n}\right)=\left\{f: \mathbf{Q}_{p} \rightarrow \mathbf{C}, f \text { is locally constant function with compact support }\right\}^{5}$.

\section{Density of Bruhat-Schwartz class in Sobolev spaces}

In the celebrated paper [30] Meyers and Serrin proved the fundamental result about density of regular functions in Sobolev spaces on $\mathbf{R}^{n}$. In [17] we have proved that $H_{\gamma}^{s}(G) \cap C(G)$ is dense in $H_{\gamma}^{s}(G)$. Nevertheless, the mentioned theorem from [17] is not enought to develop the theory of Sobolev spaces on LCA groups. Therefore, in this section we prove the density of Bruhat-Schwartz space in Sobolev spaces.

First of all, we need to recall the following crucial theorem.

Theorem 3.1. [9, 32] Let $G$ be a locally compact abelian group.

(1) The space $\mathcal{S}(G)$ is a dense subset of $L^{2}(G)$.

(2) The Fourier transform is a bijection from $\mathcal{S}(G)$ onto $\mathcal{S}\left(G^{\wedge}\right)$.

Now, we are in position to formulate and prove the main result of this section.

Theorem 3.2. If $\mathcal{S}(G) \subset H_{\gamma}^{s}(G)$, then $\mathcal{S}(G)$ is dense in $H_{\gamma}^{s}(G)$.

Remark 3.1. Let us stress that in the above theorem we do not assume metrizability of the group $G$.

\footnotetext{
${ }^{4}$ Presented examples are known in mathematical community. Although, to the best our knowledge, the proofs appear in no academic publication except for [29]. We are maintaining this tradition and we aren't also presenting the proofs.

${ }^{5}$ Complex-valued function $\psi$ is locally constant if for each point $x$ there is an integer $l(x) \in \mathbf{Z}$ such that $\psi\left(x+x^{\prime}\right)=\psi(x)$ for each $x^{\prime} \in B_{l(x)}^{n}=\left\{z:\|z\|_{p} \leq p^{l(x)}\right\}$ (for details see [3]).
} 

Since

Proof. Let us assume that $u \in H_{\gamma}^{s}(G)$ and $(u, f)_{H_{\gamma}^{s}(G)}=0$ holds for all $f \in \mathcal{S}(G)$. we get that for all $f \in \mathcal{S}(G)$

$$
(u, f)_{H_{\gamma}^{s}(G)}=\left(\mathcal{F}(u)\left(1+\gamma^{2}\right)^{s}, \mathcal{F}(f)\right)_{L^{2}\left(G^{\wedge}\right)},
$$

$$
\left(\mathcal{F}(u)\left(1+\gamma^{2}\right)^{s}, \mathcal{F}(f)\right)_{L^{2}\left(G^{\wedge}\right)}=0
$$

Moreover, because $\mathcal{F}: \mathcal{S}(G) \rightarrow \mathcal{S}\left(G^{\wedge}\right)$ is onto, we have that for all $h \in \mathcal{S}\left(G^{\wedge}\right)$

$$
\left(\mathcal{F}(u)\left(1+\gamma^{2}\right)^{s}, h\right)_{L^{2}\left(G^{\wedge}\right)}=0 .
$$

Hence, by density of $\mathcal{S}\left(G^{\wedge}\right)$ in $L^{2}\left(G^{\wedge}\right)$, we get that

$$
\mathcal{F}(u)\left(1+\gamma^{2}\right)^{s}=0,
$$

and $u=0$. This finishes the proof.

3.1. Admissible weights. In Theorem 3.2 we have seen that the sufficient condition for density of $\mathcal{S}(G)$ in $H_{\gamma}^{s}(G)$ is the inclusion $\mathcal{S}(G) \subset H_{\gamma}^{s}(G)$. Therefore we will study the class of weights $\gamma$ for which the inclusion $\mathcal{S}(G) \subset H_{\gamma}^{s}(G)$ holds. Let us start our journey with the following counterexample.

Example 4. Let us consider $H_{\gamma_{s}}^{s}\left(\mathbf{R}^{n}\right)$ with $\gamma_{s}(\xi)=\exp \left(\frac{\pi\|\xi\|^{2}}{s}\right)$. Then the function $\varphi(x)=\exp \left(-\pi\|x\|^{2}\right)$ is in Schwartz class $\mathcal{S}\left(\mathbf{R}^{n}\right)$ but does not belong to the Sobolev space $H_{\gamma_{s}}^{s}\left(\mathbf{R}^{n}\right)$. Indeed, the Fourier transform of $\varphi$ is

$$
\hat{\varphi}(\xi)=\exp \left(-\pi\|\xi\|^{2}\right)
$$

Therefore, $\gamma_{s}^{s}(\xi) \hat{\varphi}(\xi)=1$, which clearly does not belong to $L^{2}\left(\mathbf{R}^{n}\right)$. Thus, $\varphi$ is not an element of the Sobolev space $H_{\gamma_{s}}^{s}\left(\mathbf{R}^{n}\right)$.

The example shows that the weight can not grow too fast at "infinity". Now, assuming that the weight is sublinear, we prove that the Bruhat-Schwartz class is contained in the Sobolev space.

Proposition 3.1. Let $G$ be an LCA group. Suppose that $\gamma: G^{\wedge} \rightarrow[0,+\infty)$ is bounded on compact sets and is subadditive i.e. for each $\xi, \eta \in G^{\wedge}$ we have

$$
\gamma(\xi \eta) \leq \gamma(\xi)+\gamma(\eta)
$$

Then for any $s>0$ the inclusion holds $\mathcal{S}(G) \subset H_{\gamma}^{s}(G)$.

Proof. Let us take $f \in \mathcal{S}(G)$ and let $C \subset G^{\wedge}$ be a compact set from the definition of $\hat{f} \in \mathcal{A}\left(G^{\wedge}\right)$. Since $\gamma$ is bounded on compact sets, there exists a constant $c \geq 1$ such that

$$
\gamma(\xi) \leq c \quad \text { for all } \xi \in C
$$

From the subadditivity of $\gamma$ follows that for $\xi, \eta \in C$

$$
\gamma(\xi \eta) \leq 2 c
$$

Therefore, by induction for $\xi \in C^{k}$ we get that

$$
\gamma(\xi) \leq k c \text {. }
$$

We have

$$
\begin{aligned}
& \int_{G^{\wedge}}\left(1+\gamma^{2}(\xi)\right)^{s}|\hat{f}(\xi)|^{2} d \hat{\mu}_{G}(\xi) \\
& =\int_{G^{\wedge} \backslash C}\left(1+\gamma^{2}(\xi)\right)^{s}|\hat{f}(\xi)|^{2} d \hat{\mu}_{G}(\xi)+\int_{C}\left(1+\gamma^{2}(\xi)\right)^{s}|\hat{f}(\xi)|^{2} d \hat{\mu}_{G}(\xi)=I_{1}+I_{2} .
\end{aligned}
$$


The second integral $I_{2}$ can be estimated as follows

$$
I_{2}=\int_{C}\left(1+\gamma^{2}(\xi)\right)^{s}|\hat{f}(\xi)|^{2} d \hat{\mu}_{G}(\xi) \leq\left(1+c^{2}\right)^{s}\|\hat{f}\|_{L^{\infty}\left(G^{\wedge}\right)}^{2} \hat{\mu}_{G}(C)<\infty .
$$

Since $\hat{f}=0$ a.e. on $G^{\wedge} \backslash\langle C\rangle$, by Remark 2.3 we can rewrite $I_{1}$ as follows

$$
I_{1}=\sum_{k=1}^{\infty} \int_{C^{k+1} \backslash C^{k}}\left(1+\gamma^{2}(\xi)\right)^{s}|\hat{f}(\xi)|^{2} d \hat{\mu}_{G}(\xi) .
$$

Lemma 2.3, inequality (2) and Corollary 2.2 allow us to estimate the integrals in the following manner

$$
\begin{aligned}
& \int_{C^{k+1} \backslash C^{k}}\left(1+\gamma^{2}(\xi)\right)^{s}|\hat{f}(\xi)|^{2} d \hat{\mu}_{G}(\xi) \\
& \leq\left(1+(k+1)^{2} c^{2}\right)^{s}\|\hat{f}\|_{L^{\infty}\left(G^{\wedge}\right)} \int_{C^{k+1} \backslash C^{k}}|\hat{f}(\xi)| d \hat{\mu}_{G}(\xi) \\
& \leq\left(1+(k+1)^{2} c^{2}\right)^{s}\|\hat{f}\|_{L^{\infty}\left(G^{\wedge}\right)} M_{n}^{\prime} k^{-n} \\
& \leq 2^{3 s} c^{2 s} M_{n}^{\prime}\|\hat{f}\|_{L^{\infty}\left(G^{\wedge}\right)} k^{2 s-n},
\end{aligned}
$$

where $n$ can be chosen arbitrarily large. Thus, taking $n>2 s+1$, we get

$$
I_{1}=\sum_{k=1}^{\infty} \int_{C^{k+1} \backslash C^{k}}\left(1+\gamma^{2}(\xi)\right)^{s}|\hat{f}(\xi)|^{2} d \hat{\mu}_{G}(\xi) \leq 2^{3 s} c^{2 s} M_{n}^{\prime}\|\hat{f}\|_{L^{\infty}\left(G^{\wedge}\right)} \sum_{k=1}^{\infty} k^{2 s-n}<+\infty
$$

which ends the proof.

Remark 3.2. In fact, we have proved that

$$
\left\{f \in L^{2}(G): \hat{f} \in \mathcal{A}\left(G^{\wedge}\right)\right\} \subset H_{\gamma}^{s}(G) .
$$

As a corollary we obtain the following result.

Proposition 3.2. Let $G$ be a locally compact abelian group and $s \geq 0$. Suppose that there exists an invariant metric $\hat{d}$ on $G^{\wedge}$ and define $\gamma(\xi)=\hat{d}(\xi, \hat{e})$. Then $\mathcal{S}(G) \subset$ $H_{\gamma}^{s}(G)$.

Proof. Due to Proposition 3.1, it is enough to prove that $\gamma(\xi)=\hat{d}(\xi, \hat{e})$ is subadditive. Since $\hat{d}$ is invariant, we obtain

$$
\begin{aligned}
\gamma(\xi \eta) & =\hat{d}(\xi \eta, \hat{e})=\hat{d}\left(\eta, \xi^{-1}\right) \\
& \leq \hat{d}(\eta, \hat{e})+\hat{d}\left(\hat{e}, \xi^{-1}\right)=\hat{d}(\eta, \hat{e})+\hat{d}(\xi, \hat{e})=\gamma(\eta)+\gamma(\xi),
\end{aligned}
$$

and the proof follows.

Proposition 3.3. Let $G$ be an LCA group. Suppose that $\gamma: G^{\wedge} \rightarrow[0,+\infty)$ is such that for each compact set $C \subset G^{\wedge}$ there exists $\beta>0$ and $M \geq 1$ such that for each number $k \geq 0$ we have

$$
\left\|\left.\gamma\right|_{C^{k}}\right\|_{L^{\infty}(G)} \leq(M k)^{\beta}
$$

Then $\mathcal{S}(G) \subset H_{\gamma}^{s}(G)$ for each $s \geq 0$. 
Proof. Let $\varphi \in \mathcal{S}(G)$ and let $C \subset G^{\wedge}$ be a compact set from the definition of $\mathcal{A}\left(G^{\wedge}\right)$ for $\hat{\varphi}$. Let us fix $n>2 s \beta+1$, then from Lemma 2.3 we have

$$
\begin{aligned}
& \int_{G^{\wedge}}\left(1+\gamma^{2}(\xi)\right)^{s}|\hat{\varphi}(\xi)|^{2} d \hat{\mu}_{G}(\xi) \\
& =\int_{C}\left(1+\gamma^{2}(\xi)\right)^{s}|\hat{\varphi}(\xi)|^{2} d \hat{\mu}_{G}(\xi)+\sum_{k=1}^{+\infty} \int_{C^{k+1} \backslash C^{k}}\left(1+\gamma^{2}(\xi)\right)^{s}|\hat{\varphi}(\xi)|^{2} d \hat{\mu}_{G}(\xi) \\
& \leq\left(1+M^{2 \beta}\right)^{s} \int_{G^{\wedge}}|\hat{\varphi}(\xi)|^{2} d \hat{\mu}_{G}(\xi) \\
& \quad+\sum_{k=1}^{+\infty}\left(1+M^{2 \beta}(k+1)^{2 \beta}\right)^{s}\|\hat{\varphi}\|_{\infty} \int_{C^{k+1} \backslash C^{k}}|\hat{\varphi}(\xi)| d \hat{\mu}_{G}(\xi) \\
& \leq\left(1+M^{2 \beta}\right)^{s}\|\varphi\|_{2}^{2}+2^{s} M^{2 s \beta}\|\hat{\varphi}\|_{\infty} \sum_{k=1}^{+\infty}(2 k)^{2 s \beta} M_{n}^{\prime} k^{-n} \\
& =\left(1+M^{2 \beta}\right)^{s}\|\varphi\|_{2}^{2}+2^{s(1+2 \beta)} M^{2 s \beta} M_{n}^{\prime}\|\hat{\varphi}\|_{\infty} \sum_{k=1}^{+\infty} k^{2 s \beta-n}<\infty
\end{aligned}
$$

and this proves that $\varphi \in H_{\gamma}^{s}(G)$.

\section{Bruhat-Schwartz class on cartesian product of groups}

Let $G$ and $H$ be locally compact abelian groups. We will study the properties of the spaces $\mathcal{A}(G \times H)$ and $\mathcal{S}(G \times H)$. Let $f: G \times H \rightarrow \mathbf{C}$ and let us fix $x \in G$. We denote by $f_{x}: H \rightarrow \mathbf{C}$ the following function

$$
f_{x}(y)=f(x, y) \quad \text { for all } y \in H .
$$

Let us recall some basic properties of multiplication of compact sets (see [32]), which will be needed in the proof of the next theorem.

Lemma 4.1. Let $G$ be a locally compact abelian group with Haar measure $\mu$. If $C$ is a compact set containing an open neighbourhood of the group unit, then

(a) there exist a natural number $m$ and points $x_{1}, \ldots, x_{m} \in G$ such that

$$
C^{2} \subset x_{1} C \cup \ldots x_{m} C,
$$

(b) there exists a natural number $m$ such that for each $k \geq 1$ the inequality holds

$$
\mu\left(C^{k}\right) \leq k^{m} \mu_{G}(C) .
$$

Now, we are in position to formulate and prove the properties of restriction for functions from the space $\mathcal{A}(G \times H)$.

Theorem 4.1. Let $G$ and $H$ be LCA groups, then:

(i) If $f \in C(G \times H)$, then $f_{x} \in C(H)$.

(ii) If $f \in \mathcal{A}(G \times H)$, then $f_{x} \in \mathcal{A}(H)$ for almost all $x \in G$.

(iii) If $f \in \mathcal{A}(G \times H) \cap C(G \times H)$ and $g(x)=\int_{H} f_{x}(y) d \mu_{H}(y)$, then $g \in \mathcal{A}(G) \cap$ $C(G)$.

Proof. Let us denote by $\pi_{G}$ and $\pi_{H}$ the projections of $G \times H$ onto $G$ and $H$ respectively. If $C(f)$ is a compact set from the definition of $\mathcal{A}(G \times H)$, then $C(f) \subset \pi_{G}(C(f)) \times \pi_{H}(C(f))$. Therefore, we can assume that $C(f)=A \times B$ for 
some compact sets $A$ and $B$. We can also enlarge those sets if needed so they contain an open neighbourhood of the group unit.

(i) The continuity of $f_{x}$ is obvious.

(ii) From the definition of class $\mathcal{A}(G \times H)$, we have that for all $n$ there exists $M_{n}$ such that for all $k$ the following inequality holds

$$
\left\|\left.f\right|_{G \times H \backslash A^{k} \times B^{k}}\right\|_{L^{\infty}(G \times H)} \leq M_{n} k^{-n} .
$$

In particular, we obtain

$$
\left\|\left.f\right|_{G \times\left(H \backslash B^{k}\right)}\right\|_{L^{\infty}(G \times H)} \leq M_{n} k^{-n} .
$$

Hence, by the Fubini theorem we get the existence of sets $A_{n, k} \subset G$ and $B_{n, k, x} \subset$ $H \backslash B^{k}$ with $x \in A_{n, k}$ such that $\mu_{G}\left(G \backslash A_{n, k}\right)=0, \mu_{H}\left(H \backslash B^{k} \backslash B_{n, k, x}\right)=0$ and for all $x \in A_{n, k}$ and $y \in B_{n, k, x}$ we have

$$
|f(x, y)| \leq M_{n} k^{-n}
$$

Next, we define the set $A=\bigcap_{n, k} A_{n, k}$ of full measure. Therefore, for each $x \in A$, all $k, n$ and all $y \in B_{n, k, x}$ we have

$$
|f(x, y)| \leq M_{n} k^{-n}
$$

In other words, we get that for almost all $x \in G$ and for all $n, k$

$$
\left\|\left.f(x, \cdot)\right|_{H \backslash B^{k}}\right\|_{L^{\infty}(H)} \leq M_{n} k^{-n},
$$

and from that the proof of (ii) follows.

(iii) Assume that $f \in \mathcal{A}(G \times H) \cap C(G \times H)$. From the proof of (ii) we see that $f_{x} \in \mathcal{A}(H)$ for all $x \in G$. Therefore, by Corollary 2.2 we have that $f_{x} \in L^{1}(H)$ and so for all $x \in G$

$$
|g(x)|<+\infty \text {. }
$$

First of all, we will show that $g$ is a continuous function. For this purpose we fix a point $x_{0} \in G$ and $\varepsilon>0$. Let $V \subset G$ be an open neighbourhood of $x_{0}$ such that $\bar{V}$ is a compact set. Furthermore, let $\tilde{A}:=A \cup \bar{V}$ where $A$ is a set from the proof of part (ii). Then, we have

$$
\left\|\left.f\right|_{G \times H \backslash \tilde{A}^{l} \times B^{l}}\right\|_{L^{\infty}(G \times H)} \leq \frac{1}{2} M_{n} l^{-n} .
$$

Since $f$ is continuous, from the above inequality we get in particular that for all $x \in \tilde{A}^{l}$ and all $y \in H \backslash B^{l}$

$$
|f(x, y)| \leq M_{n} l^{-n} .
$$

Since $f_{x} \in \mathcal{A}(H)$ for $x \in G$, we have $f_{x}=0$ almost everywhere outside $\langle B\rangle$. Therefore, for each fixed $k$ we have

$$
\begin{aligned}
\int_{H \backslash B^{k}}|f(x, y)| d \mu_{H}(y) & =\int_{H \backslash\langle B\rangle}|f(x, y)| d \mu_{H}(y)+\sum_{l=k}^{\infty} \int_{B^{l+1} \backslash B^{l}}|f(x, y)| d \mu_{H}(y) \\
& =\sum_{l=k}^{\infty} \int_{B^{l+1} \backslash B^{l}}|f(x, y)| d \mu_{H}(y) .
\end{aligned}
$$

By Lemma 4.1, we get that there exists $m_{0}$ such that for all $l \geq k$ and each $x \in \tilde{A}^{k}$ the following inequality holds

$$
\begin{aligned}
\int_{B^{l+1} \backslash B^{l}}|f(x, y)| d \mu_{H}(y) & \leq M_{n} l^{-n} \mu_{H}\left(B^{l+1}\right) \leq M_{n} l^{-n}(l+1)^{m_{0}} \mu_{H}(B) \\
& \leq 2^{m_{0}} M_{n} \mu(B) l^{m_{0}-n}
\end{aligned}
$$


Next, let us take $n=m_{0}+2+N$, then

$\int_{H \backslash B^{k}}|f(x, y)| d \mu_{H}(y) \leq \sum_{l=k}^{\infty} 2^{m_{0}} M_{m_{0}+2+N} \mu(B) l^{-2-N} \leq 2^{m_{0}} M_{m_{0}+2+N} \mu(B) k^{-N} \sum_{l=k}^{\infty} l^{-2}$.

Denoting $M_{N}^{\prime}=2^{m_{0}} M_{2+N+m_{0}} \mu(B) \sum_{l=1}^{\infty} l^{-2}$, the above inequality takes the following form

$$
\int_{H \backslash B^{k}}|f(x, y)| d \mu_{H}(y) \leq M_{N}^{\prime} k^{-N}
$$

for all $k, N$ and any $x \in \tilde{A}^{k}$. Subsequently, we fix $N=1$ and taking $k$ large enough, we get that for all $x \in \tilde{A}^{k}$

$$
\int_{H \backslash B^{k}}|f(x, y)| d \mu_{H}(y) \leq \frac{\varepsilon}{4} .
$$

Furthermore, since $f$ is a continuous function on a compact set $\bar{V} \times B^{k}$, it is uniformly continuous. It follows that there exists an open neighbourhood $U_{x_{0}}$ of $x_{0}$ such that for all $y \in B^{k}$ and all $x \in U_{x_{0}}$ we have

$$
\left|f(x, y)-f\left(x_{0}, y\right)\right| \leq \varepsilon / 2 \mu\left(B^{k}\right) .
$$

Therefore, gathering (3) with (4), we get for $x \in U_{x_{0}} \subset A^{k}$ the following inequality

$$
\begin{aligned}
\left|g(x)-g\left(x_{0}\right)\right| & \leq \int_{H \backslash B^{k}}|f(x, y)|+\left|f\left(x_{0}, y\right)\right| d \mu_{H}(y)+\int_{B^{k}}\left|f(x, y)-f\left(x_{0}, y\right)\right| d \mu_{H}(y) \\
& \leq 2 \varepsilon / 4+\mu\left(B^{k}\right) \cdot \varepsilon / 2 \mu\left(B^{k}\right)=\varepsilon,
\end{aligned}
$$

which completes the proof of continuity of $g$.

Next, we will show that $g \in \mathcal{A}(G)$. By assumptions, for all $n$ there exists $M_{n}>0$ such that for all $k$

Therefore, we obtain

$$
\left\|\left.f\right|_{G \times H \backslash A^{k} \times B^{k}}\right\|_{L^{\infty}(G \times H)} \leq \frac{1}{2} M_{n} k^{-n} .
$$

$$
|f(x, y)| \leq M_{n} k^{-n} \quad \text { for }(x, y) \in\left(G \backslash A^{k}\right) \times H
$$

and

$$
|f(x, y)| \leq M_{n} k^{-n} \quad \text { for }(x, y) \in G \times\left(H \backslash B^{k}\right) .
$$

Reasoning just like in the proof of continuity of $g$, by inequality (6), we get that for all $n$ there exists a constant $M_{n}^{\prime}$ such that for all $k$ and all $x \in G$

$$
\int_{H \backslash B^{k}}|f(x, y)| d \mu_{H}(y) \leq M_{n}^{\prime} k^{-n} .
$$

Let us recall that by Lemma 4.1 there exists a constant $n_{0}$ such that for all $k$

$$
\mu\left(B^{k}\right) \leq k^{n_{0}} \mu(B) .
$$

Therefore, gathering the above inequality with estimates (5) and (7), we obtain for all $x \in G \backslash A^{k}$ the following inequality

$$
\begin{aligned}
|g(x)| & \leq \int_{H \backslash B^{k}}|f(x, y)| d \mu_{H}(y)+\int_{B^{k}}|f(x, y)| d \mu_{H}(y) \leq M_{n}^{\prime} k^{-n}+\mu(B) k^{n_{0}} M_{n} k^{-n} \\
& \leq\left(M_{n}^{\prime}+\mu(B) M_{n}\right) k^{n_{0}-n} .
\end{aligned}
$$

From this, we obtain that $g \in \mathcal{A}(G)$ and the whole proof follows. 
Next, we formulate and prove the properties of restrictions from the BruhatSchwartz space $\mathcal{S}(G \times H)$.

Theorem 4.2. Let $G$ and $H$ be $L C A$ groups. If $f \in \mathcal{S}(G \times H)$, then

(a) If $\xi \in G^{\wedge}$ and $g(y):=\hat{f}_{y}(\xi)$, then $g \in \mathcal{S}(H)$.

(b) For all $y \in H$ and $\xi \in G^{\wedge}$ the equality holds

$$
\hat{f}_{y}(\xi)=\int_{H^{\wedge}} \eta(y) \hat{f}(\xi, \eta) d \hat{\mu}_{H}(\eta) .
$$

(c) If $y \in H$, then $f_{y} \in \mathcal{S}(G)$.

Proof. (a) Since each $\xi \in G^{\wedge}$ is a bounded continuous function, it follows that multiplication of $f$ by such a function is still in $\mathcal{A}(G \times H) \cap C(G \times H)$. Therefore, by virtue of Theorem 4.1 we get that

$$
g(y)=\hat{f}_{y}(\xi)=\int_{G} \overline{\xi(x)} f(x, y) d \mu_{G}(x) \in \mathcal{A}(H) \cap C(H) .
$$

Furthermore, from the definition of the Fourier transform we have

$$
\begin{aligned}
\hat{f}(\xi, \eta) & =\int_{G \times H} \overline{\xi(x) \eta(y)} f(x, y) d \mu_{G \times H}(x, y)=\int_{H} \int_{G} \overline{\xi(x)} \cdot \overline{\eta(y)} f(x, y) d \mu_{G}(x) d \mu_{H}(y) \\
& =\int_{H} \overline{\eta(y)} \hat{f}_{y}(\xi) d \mu_{H}(y)=\int_{H} \overline{\eta(y)} g(y) d \mu_{H}(y)=\hat{g}(\eta) .
\end{aligned}
$$

Above equality means that $\hat{g}$ is a restriction of $\hat{f}$ to the dual group $H^{\wedge}$. Hence, since $\hat{f} \in \mathcal{A}\left(G^{\wedge} \times H^{\wedge}\right) \cap C\left(G^{\wedge} \times H^{\wedge}\right)$, by Theorem 4.1 we conclude that $\hat{g} \in \mathcal{A}\left(H^{\wedge}\right)$. Therefore, $g \in \mathcal{S}(H)$ and this finishes the proof of (a).

(b) Let us note that the Fourier inversion formula is valid for functions from the Bruhat-Schwartz class. Thus, for all $y \in H$ we have

$$
\hat{f}_{y}(\xi)=g(y)=\int_{H^{\wedge}} \eta(y) \hat{g}(\eta) d \hat{\mu}_{H}(\eta)=\int_{H^{\wedge}} \eta(y) \hat{f}(\xi, \eta) d \hat{\mu}_{H}(\eta) .
$$

(c) Since $f \in \mathcal{A}(G \times H) \cap C(G \times H)$, from Theorem 4.1 we get $f_{y} \in \mathcal{A}(G) \cap C(G)$. On the other hand, we have that the following function

$$
u(\xi, \eta):=\eta(y) \hat{f}(\xi, \eta)
$$

belongs to $\mathcal{A}\left(G^{\wedge} \times H^{\wedge}\right) \cap C\left(G^{\wedge} \times H^{\wedge}\right)$. Thus, application of Theorem 4.1 to (8) yields $\hat{f}_{y} \in \mathcal{A}(G)$, which completes the proof of Theorem 4.2.

\section{Trace of Sobolev functions}

In this section we prove the trace theorem on the product of groups. For the classical trace theorem in the Euclidean space we refer to the paper of Gagliardo [13] (see also [31, 41, 42]).

Our main result of this section is the following claim.

Theorem 5.1. Let $G, H$ be $L C A$ groups such that $H^{\wedge}$ is metrizable with a metric $\hat{d}$ and equipped with an upper $\beta$-regular measure $\hat{\mu}_{H}$. Suppose that $\gamma: G^{\wedge} \times$ $H^{\wedge} \rightarrow[0,+\infty)$ and $\tilde{\gamma}: G^{\wedge} \rightarrow[0,+\infty)$ satisfy, the following relation:

$$
\forall \xi \in G^{\wedge}, \eta \in H^{\wedge} \quad \gamma(\xi, \eta) \geq \tilde{\gamma}(\xi)+\hat{d}\left(\eta, \hat{e}_{H}\right) .
$$

If $s>\beta / 2$ and $\mathcal{S}(G \times H) \subset H_{\gamma}^{s}(G \times H)$, then there exists a linear operator

$$
T: H_{\gamma}^{s}(G \times H) \rightarrow H_{\tilde{\gamma}}^{s-\beta / 2}(G)
$$


such that

1) $\forall f \in \mathcal{S}(G \times H) \forall x \in G(T f)(x)=f\left(x, e_{H}\right)$.

2) $\forall f \in H_{\gamma}^{s}(G \times H)\|T f\|_{H_{\tilde{\gamma}}^{s-\beta / 2}(G)} \leq C\|f\|_{H_{\gamma}^{s}(G \times H)}$.

Proof. Let us take $f \in \mathcal{S}(G \times H)$ and define $g \in \mathcal{S}(G)$ as follows

$$
\forall x \in G \quad g(x)=f\left(x, e_{H}\right) .
$$

From point (b) of Theorem 4.2 we get the equality

$$
\hat{g}(\xi)=\int_{H^{\wedge}} \hat{f}(\xi, \eta) d \hat{\mu}_{H}(\eta) .
$$

Furthermore, it follows that

$$
\begin{aligned}
|\hat{g}(\xi)|^{2} & =\left|\int_{H^{\wedge}} \hat{f}(\xi, \eta) d \hat{\mu}_{H}(\eta)\right|^{2}=\left|\int_{H^{\wedge}} \hat{f}(\xi, \eta) \frac{\left(1+\gamma^{2}(\xi, \eta)\right)^{s / 2}}{\left(1+\gamma^{2}(\xi, \eta)\right)^{s / 2}} d \hat{\mu}_{H}(\eta)\right|^{2} \\
& \leq \int_{H^{\wedge}}\left(1+\gamma^{2}(\xi, \eta)\right)^{s}|\hat{f}(\xi, \eta)|^{2} d \hat{\mu}_{H}(\eta) \cdot \int_{H^{\wedge}}\left(1+\gamma^{2}(\xi, \eta)\right)^{-s} d \hat{\mu}_{H}(\eta) .
\end{aligned}
$$

Now, we claim that there exists a constant $C>0$ independent of $\xi$ such that

$$
\int_{H^{\wedge}}\left(1+\gamma^{2}(\xi, \eta)\right)^{-s} d \hat{\mu}_{H}(\eta) \leq C\left(1+\tilde{\gamma}^{2}(\xi)\right)^{\beta / 2-s} .
$$

Indeed, using assumption on $\gamma$ we have

$$
\int_{H^{\wedge}} \frac{d \hat{\mu}_{H}(\eta)}{\left(1+\gamma^{2}(\xi, \eta)\right)^{s}} \leq \int_{H^{\wedge}} \frac{d \hat{\mu}_{H}(\eta)}{\left(1+\tilde{\gamma}^{2}(\xi)+\hat{d}\left(\hat{e}_{H}, \eta\right)^{2}\right)^{s}} .
$$

From Lemma 2.1 we get that for any $r>0$ we have

$$
\int_{H^{\wedge} \backslash B\left(\hat{e}_{H}, r\right)} \frac{d \hat{\mu}_{H}(\eta)}{\left(1+\hat{d}\left(\hat{e}_{H}, \eta\right)^{2}\right)^{s}} \leq C r^{\beta-2 s} .
$$

We need to consider two cases.

1) When $H^{\wedge}$ is not discrete, then taking $r=1+\tilde{\gamma}(\xi)$ we get

$$
\begin{aligned}
& \int_{H^{\wedge}} \frac{d \hat{\mu}_{H}(\eta)}{\left(1+\tilde{\gamma}^{2}(\xi)+\hat{d}\left(\hat{e}_{H}, \eta\right)^{2}\right)^{s}} \\
& =\int_{B\left(\hat{e}_{H}, r\right)} \frac{d \hat{\mu}_{H}(\eta)}{\left(1+\tilde{\gamma}^{2}(\xi)+\hat{d}\left(\hat{e}_{H}, \eta\right)^{2}\right)^{s}}+\int_{H^{\wedge} \backslash B\left(\hat{e}_{H}, r\right)} \frac{d \hat{\mu}_{H}(\eta)}{\left(1+\tilde{\gamma}^{2}(\xi)+\hat{d}\left(\hat{e}_{H}, \eta\right)^{2}\right)^{s}} \\
& \leq \frac{1}{\left(1+\tilde{\gamma}^{2}(\xi)\right)^{s}} \hat{\mu}_{H}\left(B\left(\hat{e}_{H}, r\right)\right)+C r^{\beta-2 s} \\
& \leq D_{\beta}\left(1+\tilde{\gamma}^{2}(\xi)\right)^{-s}(1+\tilde{\gamma}(\xi))^{\beta}+C(1+\tilde{\gamma}(\xi))^{\beta-2 s} \\
& \leq 2^{\beta / 2} D_{\beta}\left(1+\tilde{\gamma}^{2}(\xi)\right)^{-s}\left(1+\tilde{\gamma}^{2}(\xi)\right)^{\beta / 2}+C 2^{\beta / 2-s}\left(1+\tilde{\gamma}^{2}(\xi)\right)^{\beta / 2-s} \\
& =\left(2^{\beta / 2} D_{\beta}+C 2^{\beta / 2-s}\right)\left(1+\tilde{\gamma}^{2}(\xi)\right)^{\beta / 2-s} .
\end{aligned}
$$

2) If $H^{\wedge}$ is discrete, then we have two possibilities.

(i) If $R_{0} \leq r=1+\tilde{\gamma}(\xi)$, then we repeat the proof for not discrete groups.

(ii) If $R_{0} \geq r=1+\tilde{\gamma}(\xi)$, then from Definition 2.3 we have

$$
\int_{B\left(\hat{e}_{H}, r\right)} \frac{d \hat{\mu}_{H}(\eta)}{\left(1+\tilde{\gamma}^{2}(\xi)+\hat{d}\left(\hat{e}_{H}, \eta\right)^{2}\right)^{s}}=\frac{1}{\left(1+\tilde{\gamma}^{2}(\xi)\right)^{s}} .
$$


Therefore we can finish the proof of (10) exploring the methods from 1). This finishes the proof of inequality (10).

Now, if we combine (9) and (10) we get

$$
|\hat{g}(\xi)|^{2} \leq C\left(1+\tilde{\gamma}^{2}(\xi)\right)^{\beta / 2-s} \cdot \int_{H^{\wedge}}\left(1+\gamma^{2}(\xi, \eta)\right)^{s}|\hat{f}(\xi, \eta)|^{2} d \hat{\mu}_{H}(\eta) .
$$

The process of multiplying the above inequality by $\left(1+\tilde{\gamma}^{2}(\xi)\right)^{s-\beta / 2}$ and integrating over $G^{\wedge}$ leads us to the following inequality

$$
\|g\|_{H_{\tilde{\gamma}}^{s-\beta / 2}(G)} \leq C\|f\|_{H_{\gamma}^{s}(G \times H)} .
$$

This shows the continuity of the linear operator $T: H_{\gamma}^{s}(G \times H) \cap \mathcal{S}(G \times H) \rightarrow$ $H_{\tilde{\gamma}}^{s-\beta / 2}(G)$.

Finally, using Theorem 3.2 we get that $\mathcal{S}(G \times H)$ is a dense subset of $H_{\gamma}^{s}(G \times$ $H)$, thus by the usual limiting argument we can extend our operator to the whole $H_{\gamma}^{s}(G \times H)$. This completes the proof of the theorem.

It is well known that the trace operator $T: H^{s}\left(\mathbf{R}^{n}\right) \rightarrow H^{s-1 / 2}\left(\mathbf{R}^{n-1}\right)$ is surjective (see e.g. $[42,31]$ ). In the next subsection we shall prove that the trace operator is surjective in the case of $p$-adic numbers. Nevertheless, it is not clear if the surjectivity of the trace operator holds for the product of any two LCA groups. Even it is not at all clear in the case of the torus $\mathbf{T}^{n}$. Hence, it is natural to state the following problem.

Open problem 1. Is $T: H^{s}\left(\mathbf{T}^{n}\right) \rightarrow H^{s-1 / 2}\left(\mathbf{T}^{n-1}\right)$ surjective?

5.1. Trace theorem on $\mathrm{Q}_{p}^{n}$. In this section we shall study the Sobolev space $H^{s}\left(\mathbf{Q}_{p}^{n}\right)$ on $p$-adic group $\mathbf{Q}_{p}^{n}[33,40]$. Let us recall that

$$
H^{s}\left(\mathbf{Q}_{p}^{n}\right)=\left\{u \in L^{2}\left(\mathbf{Q}_{p}^{n}\right): \int_{\mathbf{Q}_{p}^{n}}\left(1+\|\xi\|_{p}^{2}\right)^{s}|\hat{u}(\xi)|^{2} d \xi<\infty\right\} .
$$

Remark 5.1. There are many equivalent norms on $H^{s}\left(\mathbf{Q}_{p}^{n}\right)$. We shall use the following equivalent norms

$$
\|u\|_{1, \mathbf{Q}_{p}^{n}}^{2}=\int_{\mathbf{Q}_{p}^{n}} \max \left(1,\|\xi\|_{p}\right)^{2 s}|\hat{u}(\xi)|^{2} d \xi
$$

and

$$
\|u\|_{2, \mathbf{Q}_{p}^{n}}^{2}=\int_{\mathbf{Q}_{p}^{n-1}} \int_{\mathbf{Q}_{p}}\left(\max \left(1,\left\|\xi^{\prime}\right\|_{p}\right)+\left|\xi_{n}\right|_{p}\right)^{2 s}|\hat{u}(\xi)|^{2} d \xi_{n} d \xi^{\prime} .
$$

Our goal is to show that the Trace operator $T: H^{s}\left(\mathbf{Q}_{p}^{n}\right) \rightarrow H^{s-1 / 2}\left(\mathbf{Q}_{p}^{n-1}\right)$ constructed in the previous section is surjective.

Theorem 5.2. Assume that $n>1$ and $s>1 / 2$. Then the Trace operator

$$
T: H^{s}\left(\mathbf{Q}_{p}^{n}\right) \rightarrow H^{s-1 / 2}\left(\mathbf{Q}_{p}^{n-1}\right)
$$

is surjective.

Proof. Let us denote

$$
\omega\left(\xi^{\prime}\right):= \begin{cases}1, & \text { if }\left\|\xi^{\prime}\right\|_{p} \leq 1 \\ p^{-\gamma}, & \text { if }\left\|\xi^{\prime}\right\|_{p}=p^{\gamma} \text { for some } \gamma \geq 0 .\end{cases}
$$

Then clearly

$$
\left|\omega\left(\xi^{\prime}\right)\right|_{p}=\max \left(1,\left\|\xi^{\prime}\right\|_{p}\right)
$$


and we can write the equivalent norm on $H^{s}\left(\mathbf{Q}_{p}\right)$ in the following manner

$$
\|u\|_{2, \mathbf{Q}_{p}^{n}}^{2}=\int_{\mathbf{Q}_{p}^{n-1}} \int_{\mathbf{Q}_{p}}\left(\left|\omega\left(\xi^{\prime}\right)\right|_{p}+\left|\xi_{n}\right|_{p}\right)^{2 s}|\hat{u}(\xi)|^{2} d \xi_{n} d \xi^{\prime} .
$$

Now, in order to prove that $T$ is onto we take $v \in H^{s-\frac{1}{2}}\left(\mathbf{Q}_{p}^{n-1}\right)$ and define

$$
\tilde{u}\left(\xi^{\prime}, \xi_{n}\right):=\hat{v}\left(\xi^{\prime}\right)\left|\omega\left(\xi^{\prime}\right)\right|_{p}^{-1} \varphi\left(\frac{\xi_{n}}{\omega\left(\xi^{\prime}\right)}\right)
$$

where $\varphi \in \mathcal{S}\left(\mathbf{Q}_{p}\right)$ is a characteristic function of a unit ball $B(0,1)$ in $\mathbf{Q}_{p}$.

We claim that $\tilde{u} \in L^{2}\left(\mathbf{Q}_{p}^{n}\right)$. Indeed, by substituting $\xi_{n}=t \omega\left(\xi^{\prime}\right)$, with the Jacobian $\left|\omega\left(\xi^{\prime}\right)\right|_{p}$ we have

$$
\int_{\mathbf{Q}_{p}} \varphi^{2}\left(\frac{\xi_{n}}{\omega\left(\xi^{\prime}\right)}\right) d \xi_{n}=\left|\omega\left(\xi^{\prime}\right)\right|_{p}
$$

hence

$$
\begin{aligned}
\mid \tilde{u} \|_{L^{2}\left(\mathbf{Q}_{p}^{n}\right)}^{2} & =\int_{\mathbf{Q}_{p}^{n-1}} \int_{\mathbf{Q}_{p}}\left|\hat{v}\left(\xi^{\prime}\right)\right|^{2}\left|\omega\left(\xi^{\prime}\right)\right|_{p}^{-2} \varphi^{2}\left(\frac{\xi_{n}}{\omega\left(\xi^{\prime}\right)}\right) d \xi_{n} d \xi^{\prime} \\
& =\int_{\mathbf{Q}_{p}^{n-1}} \frac{\left|\hat{v}\left(\xi^{\prime}\right)\right|^{2}}{\left|\omega\left(\xi^{\prime}\right)\right|_{p}} d \xi^{\prime} \leq\|\hat{v}\|_{L^{2}\left(\mathbf{Q}_{p}^{n-1}\right) .}
\end{aligned}
$$

Therefore, there exists $u \in L^{2}\left(\mathbf{Q}_{p}^{n}\right)$ such that $\hat{u}=\tilde{u}$.

Now, we shall prove that $u \in H^{s}\left(\mathbf{Q}_{p}^{n}\right)$. Using the change of variable formula, we have

$$
\begin{aligned}
& \int_{\mathbf{Q}_{p}}\left(\left|\omega\left(\xi^{\prime}\right)\right|_{p}+\left|\xi_{n}\right|_{p}\right)^{2 s}\left|\hat{u}\left(\xi^{\prime}, \xi_{n}\right)\right|^{2} d \xi_{n} \\
& =\int_{\mathbf{Q}_{p}}\left(\left|\omega\left(\xi^{\prime}\right)\right|_{p}+\left|\xi_{n}\right|_{p}\right)^{2 s}\left|\hat{v}\left(\xi^{\prime}\right)\right|^{2}\left|\omega\left(\xi^{\prime}\right)\right|_{p}^{-2} \varphi^{2}\left(\frac{\xi_{n}}{\omega\left(\xi^{\prime}\right)}\right) d \xi_{n} \\
& =\int_{\mathbf{Q}_{p}}\left(\left|\omega\left(\xi^{\prime}\right)\right|_{p}+\left|t \omega\left(\xi^{\prime}\right)\right|_{p}\right)^{2 s}\left|\hat{v}\left(\xi^{\prime}\right)\right|^{2}\left|\omega\left(\xi^{\prime}\right)\right|_{p}^{-1} \varphi^{2}(t) d t \\
& =\left|\omega\left(\xi^{\prime}\right)\right|_{p}^{2 s-1}\left|\hat{v}\left(\xi^{\prime}\right)\right|_{p}^{2} \int_{\mathbf{Q}_{p}}\left(1+|t|_{p}\right)^{2 s} \varphi^{2}(t) d t \leq 2^{2 s}\left|\omega\left(\xi^{\prime}\right)\right|_{p}^{2 s-1}\left|\hat{v}\left(\xi^{\prime}\right)\right|_{p}^{2}
\end{aligned}
$$

From the above estimates, we get that

$$
\|u\|_{2, \mathbf{Q}_{p}^{n}}^{2} \leq 2^{2 s} \int_{\mathbf{Q}_{p}^{n-1}}\left|\omega\left(\xi^{\prime}\right)\right|_{p}^{2 s-1}\left|\hat{v}\left(\xi^{\prime}\right)\right|_{p}^{2} d \xi^{\prime}=2^{2 s}\|v\|_{1, \mathbf{Q}_{p}^{n-1}}^{2} .
$$

Since the last norm is equivalent to $\|v\|_{H^{s-1 / 2}\left(\mathbf{Q}_{p}^{n-1}\right)}$, we conclude that $u \in H^{s}\left(\mathbf{Q}_{p}^{n}\right)$.

Finally, we shall prove that $T u=v$. Let us observe that ${ }^{6}$

$$
\int_{\mathbf{Q}_{p}} \hat{u}\left(\xi^{\prime}, \xi_{n}\right) d \xi_{n}=\hat{v}\left(\xi^{\prime}\right)
$$

Let $f_{k} \in \mathcal{S}\left(\mathbf{Q}_{p}^{n}\right)$ be such that $f_{k} \rightarrow u$ in $H^{s}\left(\mathbf{Q}_{p}^{n}\right)$. This implies that $T f_{k} \rightarrow T u$ in $H^{s-1 / 2}\left(\mathbf{Q}_{p}^{n-1}\right)$ and in particular we have

$$
\widehat{T f_{k}} \rightarrow \widehat{T u} \quad \text { in } L^{2}\left(\mathbf{Q}_{p}^{n-1}\right) \text {. }
$$

\footnotetext{
${ }^{6}$ This follows from the identity $\int_{\mathbf{Q}_{p}} \varphi(t) d t=1$.
} 
We will prove that $\widehat{T f_{k}} \rightarrow \hat{v}$ in $L^{2}\left(\mathbf{Q}_{p}^{n-1}\right)$ which will show that $T u=v$. Since $f_{k} \in \mathcal{S}\left(\mathbf{Q}_{p}^{n}\right)$, by Theorem 4.2 we get

$$
\widehat{T f_{k}}\left(\xi^{\prime}\right)=\int_{\mathbf{Q}_{p}} \hat{f}_{k}\left(\xi^{\prime}, \xi_{n}\right) d \xi_{n} .
$$

The Schwarz inequality yields

$$
\begin{aligned}
& \left|\int_{\mathbf{Q}_{p}}\left(\hat{f}_{k}\left(\xi^{\prime}, \xi_{n}\right)-\hat{u}\left(\xi^{\prime}, \xi_{n}\right)\right) d \xi_{n}\right| \leq \int_{\mathbf{Q}_{p}}\left|\hat{f}_{k}\left(\xi^{\prime}, \xi_{n}\right)-\hat{u}\left(\xi^{\prime}, \xi_{n}\right)\right| d \xi_{n} \\
& \leq\left(\int_{\mathbf{Q}_{p}} \frac{d \xi_{n}}{\left(1+\left|\xi_{n}\right|_{p}^{2}\right)^{s}} \int_{\mathbf{Q}_{p}}\left(1+\left|\xi_{n}\right|_{p}^{2}\right)^{s}\left|\hat{f}_{k}\left(\xi^{\prime}, \xi_{n}\right)-\hat{u}\left(\xi^{\prime}, \xi_{n}\right)\right|^{2} d \xi_{n}\right)^{1 / 2} .
\end{aligned}
$$

Since $s>1 / 2$ and $\mathbf{Q}_{p}$ is 1-regular, we have that the integral

$$
C:=\int_{\mathbf{Q}_{p}} \frac{d \xi_{n}}{\left(1+\left|\xi_{n}\right|_{p}^{2}\right)^{s}}
$$

is finite. Hence, using (11) we get

$$
\begin{aligned}
\int_{\mathbf{Q}_{p}^{n-1}}\left|\widehat{T f_{k}}\left(\xi^{\prime}\right)-\hat{v}\left(\xi^{\prime}\right)\right|^{2} d \xi^{\prime} & =\int_{\mathbf{Q}_{p}^{n-1}}\left|\int_{\mathbf{Q}_{p}}\left(\hat{f}_{k}\left(\xi^{\prime}, \xi_{n}\right)-\hat{u}\left(\xi^{\prime}, \xi_{n}\right)\right) d \xi_{n}\right|^{2} d \xi^{\prime} \\
& \leq \int_{\mathbf{Q}_{p}^{n-1}}\left(C \int_{\mathbf{Q}_{p}}\left(1+\left|\xi_{n}\right|_{p}^{2}\right)^{s}\left|\hat{f}_{k}\left(\xi^{\prime}, \xi_{n}\right)-\hat{u}\left(\xi^{\prime}, \xi_{n}\right)\right|^{2} d \xi_{n}\right) d \xi^{\prime} \\
& \leq C\left\|f_{k}-u\right\|_{H^{s}\left(\mathbf{Q}_{p}^{n}\right)}^{2}
\end{aligned}
$$

which completes the proof.

\section{Sobolev versus Aronszajn-Gagliardo-Slobodecki}

In this section we deal with fractional Sobolev spaces on LCA grups equipped with a $\beta$-regular Haar measure. We study their connections with Sobolev spaces.

Definition 6.1. Let $G$ be an LCA group with a metric $d$ and let $s \in(0,1)$. We shall say that $u \in L^{2}(G)$ belongs to the fractional Sobolev space $\mathcal{H}_{s, \beta}(G)$ if the following Slobodecki seminorm

$$
[u]_{s, \beta}^{2}=\int_{G} \int_{G} \frac{|u(x)-u(y)|^{2}}{d(x, y)^{\beta+2 s}} d \mu_{G}(x) d \mu_{G}(y)
$$

is finite. Subsequently, we define the norm in the following way

$$
\|u\|_{\mathcal{H}_{s, \beta}}=\|u\|_{L^{2}(G)}+[u]_{s, \beta} \text {. }
$$

It is an easy exercise from functional analysis to show that $\mathcal{H}_{s, \beta}(G)$ is a $\mathrm{Ba}$ nach space. On domains of $\mathbf{R}^{n}$ the above space has been introduced by Aronszajn, Gagliardo and Slobodecki (see [4, 14, 38]) and in the literature is also called Aronszajn-Gagliardo-Slobodecki space. The definition of fractional Sobolev space uses only the existence of a metric and a measure on LCA group. In order to get some deeper results we will also require existence of a dual metric.

Theorem 6.1. Let $G$ be a non-discrete LCA group such that $d$ and $\hat{d}$ are dual metrics. We assume that $d$ is an invariant metric and $\mu$ is an upper $\beta$-regular measure. If $s \in(0,1)$ and $\gamma=\hat{d}$, then

$$
H_{\gamma}^{s}(G) \hookrightarrow \mathcal{H}_{s, \beta}(G)
$$


Moreover, there exists $C>0$ such that the following inequality holds

$$
\|u\|_{\mathcal{H}_{s, \beta}(G)} \leq C\|u\|_{H_{\gamma}^{s}(G)}
$$

for each $u \in H_{\gamma}^{s}(G)$.

Proof. Let us take $u \in H_{\gamma}^{s}(G)$. From the definition of a fractional Sobolev seminorm we have

$$
[u]_{s, \beta}^{2}=\int_{G} \int_{G} \frac{|u(x)-u(y)|^{2}}{d(x, y)^{\beta+2 s}} d \mu_{G}(x) d \mu_{G}(y) .
$$

Since $d$ is invariant and $G$ abelian, by substituting $z=x-y$ and by the Fubini theorem, we get

$$
[u]_{s, \beta}^{2}=\int_{G} \int_{G} \frac{|u(z+y)-u(y)|^{2}}{d(z, e)^{\beta+2 s}} d \mu_{G}(y) d \mu_{G}(z) .
$$

We claim that

$$
[u]_{s, \beta}^{2}=\int_{G^{\wedge}}|\hat{u}(\xi)|^{2} A(\xi) d \hat{\mu}_{G}(\xi)
$$

where

$$
A(\xi)=\int_{G} \frac{|\xi(z)-1|^{2}}{d(z, e)^{\beta+2 s}} d \mu_{G}(z) .
$$

Indeed, by virtue of the Plancherel theorem we have

$$
\begin{aligned}
{[u]_{s, \beta}^{2} } & =\int_{G} \int_{G}\left|\frac{u(z+y)-u(y)}{d(z, e)^{\beta / 2+s}}\right|^{2} d \mu_{G}(y) d \mu_{G}(z)=\int_{G}\left\|\frac{u(z+\cdot)-u(\cdot)}{d(z, e)^{\beta / 2+s}}\right\|_{L^{2}(G)}^{2} d \mu_{G}(z) \\
& =\int_{G}\left\|\mathcal{F}\left(\frac{u(z+\cdot)-u(\cdot)}{d(z, e)^{\beta / 2+s}}\right)\right\|_{L^{2}\left(G^{\wedge}\right)}^{2} d \mu_{G}(z)=\int_{G}\left\|\frac{\xi(z) \hat{u}(\xi)-\hat{u}(\xi)}{d(z, e)^{\beta / 2+s}}\right\|_{L^{2}\left(G^{\wedge}\right)}^{2} d \mu_{G}(z) \\
& =\int_{G} \int_{G^{\wedge}} \frac{|\xi(z)-1|^{2}}{d(z, e)^{\beta+2 s}}|\hat{u}(\xi)|^{2} d \hat{\mu}_{G}(\xi) d \mu_{G}(z)=\int_{G^{\wedge}}|\hat{u}(\xi)|^{2} A(\xi) d \hat{\mu}_{G}(\xi) .
\end{aligned}
$$

Our next goal is to prove the existence of $C$ such that

$$
A(\xi) \leq C \hat{d}(\xi, \hat{e})^{2 s}
$$

Let $r>0$, then

$$
\begin{aligned}
A(\xi) & =\int_{B\left(e, \frac{1}{r}\right)} \frac{|\xi(z)-1|^{2}}{d(z, e)^{\beta+2 s}} d \mu_{G}(z)+\int_{G \backslash B\left(e, \frac{1}{r}\right)} \frac{|\xi(z)-1|^{2}}{d(z, e)^{\beta+2 s}} d \mu_{G}(z) \\
& \leq \int_{B\left(e, \frac{1}{r}\right)} \frac{\hat{d}(\xi, \hat{e})^{2} d(z, e)^{2}}{d(z, e)^{\beta+2 s}} d \mu_{G}(z)+4 \int_{G \backslash B\left(e, \frac{1}{r}\right)} \frac{1}{d(z, e)^{\beta+2 s}} d \mu_{G}(z)=I_{1}+4 I_{2} .
\end{aligned}
$$


Taking $r=\hat{d}(\xi, \hat{e})$ and using $\beta$-regularity, we estimate $I_{1}$ and $I_{2}$ as follows:

$$
\begin{aligned}
I_{1} & \leq \hat{d}(\xi, \hat{e})^{2} \sum_{i=1}^{\infty} \int_{B\left(e, \frac{1}{2^{i} r}\right) \backslash B\left(e, \frac{1}{2^{i+1} r}\right)} \frac{d(z, e)^{2}}{d(z, e)^{\beta+2 s}} d \mu_{G}(z) \\
& \leq \hat{d}(\xi, \hat{e})^{2} \sum_{i=1}^{\infty}\left(\frac{1}{2^{i} r}\right)^{2}\left(2^{i+1} r\right)^{\beta+2 s} C_{\beta}\left(\frac{1}{2^{i} r}\right)^{\beta}=C_{\beta} 2^{\beta+2 s} \hat{d}(\xi, \hat{e})^{2 s} \frac{1}{1-2^{-2+2 s}} . \\
I_{2} & \leq \sum_{i=1}^{\infty} \int_{B\left(e, 2^{i+1} r^{-1}\right) \backslash B\left(e, 2^{i} r^{-1}\right)} \frac{1}{d(z, e)^{\beta+2 s}} d \mu_{G}(z) \leq \sum_{i=1}^{\infty} C_{\beta}\left(2^{i+1} r^{-1}\right)^{\beta}\left(2^{i} r^{-1}\right)^{-\beta-2 s} \\
& =C_{\beta} 2^{\beta} \hat{d}(\xi, \hat{e})^{2 s} \frac{1}{1-2^{-2 s}} .
\end{aligned}
$$

This finishes the proof of (13). Hence, we conclude that

$$
[u]_{s, \beta}^{2} \leq C \int_{G^{\wedge}} \hat{d}(\xi, \hat{e})^{2 s}|\hat{u}(\xi)|^{2} d \hat{\mu}_{G}(\xi) .
$$

Furthermore, since $\gamma=\hat{d}$ we see that

$$
[u]_{s, \beta}^{2} \leq C \int_{G^{\wedge}} \gamma(\xi)^{2 s}|\hat{u}(\xi)|^{2} d \hat{\mu}_{G}(\xi)
$$

and this proves the theorem.

6.1. Examples. In this subsection we provide examples of topological groups for which Sobolev space and fractional Sobolev class coincide. We start with a very natural example, namely with the cartesian product of the $n$-dimensional euclidean space $\mathbf{R}^{n}$ with the $m$-dimensional torus $\mathbf{T}^{m}$.

Theorem 6.2. Let $G=\mathbf{R}^{n} \times \mathbf{T}^{m}$, then for $s \in(0,1)$

$$
\mathcal{H}_{s, l}(G) \simeq H_{\hat{d}}^{s}(G),{ }^{7}
$$

where $l=n+m$ and $\hat{d}$ is a canonical metric on the dual group $G^{\wedge}$.

Remark 6.1. If it is well known (see [27, C Theorem]) that any abelian connected Lie group $G$ of dimension $l$ has the following form

$$
G=\mathbf{R}^{n} \times \mathbf{T}^{m}
$$

for some $n, m \geq 0$ such that $n+m=l$. Thus, from the above theorem follows that $\mathcal{H}_{s, l}(G) \simeq H^{s}(G)$, where $l=n+m$.

Proof. If $m=0$, then the theorem is well known in the literature (see e.g. [31]), if $n=0$, then Theorem 6.2 has been proved in [7].

Since for $G=\mathbf{R}^{n} \times \mathbf{T}^{m}$ the assumptions of Theorem 6.1 are satisfied, we have $H_{\gamma}^{s}(G) \hookrightarrow \mathcal{H}_{s, n+m}(G)$. Hence, we need to show that there exists a positive constant $C$ depending only on dimensions $n$ and $m$ such that

$$
\|u\|_{H^{s}\left(\mathbf{R}^{n} \times \mathbf{T}^{m}\right)} \leq C\|u\|_{\mathcal{H}_{s, n+m}\left(\mathbf{R}^{n} \times \mathbf{T}^{m}\right)} .
$$

Let us stress that the quantity $A$ introduced in the proof of Theorem 6.1 has the following form

$$
A(\xi, k)=\int_{(-1,1]^{m}} \int_{\mathbf{R}^{n}} \frac{\sin ^{2} \pi(\xi x+k y)}{\|x\|^{2 s+n+m}+\|y\|^{2 s+n+m}} d^{n} x d^{m} y,
$$

where $\xi \in \mathbf{R}^{n}$ and $k \in \mathbf{Z}^{m}$.

\footnotetext{
${ }^{7}$ This equivalence means equality of sets and equivalence of corresponding norms.
} 
We shall show that there exists $\widetilde{C}$ such that for $(\xi, k) \in \mathbf{R}^{n} \times \mathbf{Z}^{m} \backslash \mathbf{R}^{n} \times\{0\}$

$$
A(\xi, k) \geq \widetilde{C}\left(\|\xi\|^{2 s}+\|k\|^{2 s}\right) .
$$

For this purpose we define the following quantity

$$
M:=\max \left(\max _{1 \leq i \leq n}\left|\xi_{i}\right|, \max _{1 \leq j \leq m}\left|k_{j}\right|\right) .
$$

Now we can assume without loss of generality that $\xi=\left(\xi_{1}, \ldots, \xi_{n}\right), k=\left(k_{1}, \ldots, k_{m}\right)$, where $\xi_{i} \geq 0$ for $1 \leq i \leq p$ and $k_{j} \geq 0$ for $1 \leq j \leq q$, and other position of those vectors are negative. Then we can estimate from below $A(\xi, k)$ by

$$
\int \ldots \int \frac{\sin ^{2} \pi(\xi x+k y)}{\|x\|^{2 s+n+m}+\|y\|^{2 s+n+m}} d x_{n} \ldots d x_{1} d y_{m} \ldots d y_{1}
$$

where

$$
D=\left[\frac{1}{A}, \frac{3}{A}\right]^{q} \times\left[\frac{-3}{A}, \frac{-1}{A}\right]^{m-q} \times\left[\frac{1}{A}, \frac{3}{A}\right]^{p} \times\left[\frac{-3}{A}, \frac{-1}{A}\right]^{n-p},
$$

and $A=4 M(m+n)$.

On the set $D$ we have

$$
\frac{1}{4(m+n)} \leq \xi x+k y \leq \frac{3}{4}
$$

Indeed, let us write

$$
\begin{aligned}
\xi x+k y= & \left(\xi_{1} x_{1}+\cdots+\xi_{p} x_{p}+k_{1} y_{1}+\cdots+k_{q} y_{q}\right) \\
& +\left(\xi_{p+1} x_{p+1}+\cdots+\xi_{n} x_{n}+k_{q+1} y_{q+1}+\cdots+k_{m} y_{m}\right)
\end{aligned}
$$

and notice that all ingredients in the first bracket are non-negative since all ingredients of the sum are factors of two non-negative numbers, and all components of the second bracket are non-negative since each product is a factor of negative numbers by assumption of numeration of coordinates of $\xi$ and $k$. It follows that from nonnegativity of each ingredient that $\xi x+k y$ is bounded from below by any $\xi_{i} x_{i}$ and $k_{j} y_{j}$ for any $i=1, \ldots, n$ and $j=1, \ldots, m$. From the choice of $M$ there exists $i$ or $j$ such that $\left|\xi_{i}\right|=M$ or $\left|k_{j}\right|=M$. It follows that $\xi_{i}=M$ or $\xi_{i}=-M$ or $k_{j}=M$ or $k_{j}=-M$, so

$$
\xi x+k y \geq \begin{cases}M y_{j}, & \text { if } k_{j} \geq 0, \\ -M y_{j}, & \text { if } k_{j}<0, \\ M x_{i}, & \text { if } \xi_{i} \geq 0, \\ -M x_{i}, & \text { if } \xi_{i}<0 .\end{cases}
$$

Each term on the right side of this inequality we can estimate from below by $1 / 4(m+n)$. To estimate $\xi x+k y$ from above we observe that

$$
\xi x+k y \leq M(m+n) \frac{3}{4 M(m+n)}=\frac{3}{4} .
$$

Therefore, from (16) we get

$$
\sin ^{2}(\pi(\xi x+k y)) \geq \sin ^{2} \frac{\pi}{4(m+n)}=: c_{0}>0 .
$$

Hence, we can bound $A$ as follows

$$
A(\xi, k) \geq c_{0} \int_{D} \ldots \int \frac{d x_{n} \ldots d x_{1} d y_{m} \ldots d y_{1}}{\|x\|^{2 s+n+m}+\|y\|^{2 s+n+m}}
$$


Next, by changing variables $M(n+m) x_{i}=z_{i}, M(n+m) y_{i}=t_{i}$ we get that the RHS of the above inequality is equal to

$$
c_{0}(m+n)^{2 s} M^{2 s} \int_{\left[\frac{1}{4}, \frac{3}{4}\right]^{m}} \int_{\left[\frac{1}{4}, \frac{3}{4}\right]^{n}} \frac{d^{n} z d^{m} t}{\|z\|^{2 s+n+m}+\|t\|^{2 s+n+m}}=C_{1} M^{2 s},
$$

where

$$
C_{1}=c_{0}(m+n)^{2 s} \int_{\left[\frac{1}{4}, \frac{3}{4}\right]^{m}} \int_{\left[\frac{1}{4}, \frac{3}{4}\right]^{n}} \frac{d^{n} z d^{m} t}{\|z\|^{2 s+n+m}+\|t\|^{2 s+n+m}} .
$$

Moreover, from the definition of $M$ we have

$$
M^{2 s} \geq \frac{1}{\left(n^{s}+m^{s}\right)}\left(\|\xi\|^{2 s}+\|k\|^{2 s}\right) .
$$

Thus, inequality (15) follows with $\widetilde{C}=\frac{C_{1}}{n^{s}+m^{s}}$.

Subsequently, we consider the case $k=0, \xi \in \mathbf{R}^{n}$. We have the following string of inequalities

$$
\begin{aligned}
A(\xi, 0) & \geq \int_{[-1,1]^{m}} \int_{\|x\| \leq 1} \frac{\sin ^{2}(\pi \xi x)}{\|x\|^{2 s+n+m}+\|y\|^{2 s+n+m}} d^{n} x d^{m} y \\
& =\int_{\|x\| \leq 1} \int_{[-1,1]^{m}} \frac{\sin ^{2}(\pi \xi x)}{\|x\|^{2 s+n+m}+\|y\|^{2 s+n+m}} d^{m} y d^{n} x \\
& \geq \int_{\|x\| \leq 1} \int_{\|y\| \leq\|x\|} \frac{\sin ^{2}(\pi \xi x)}{\|x\|^{2 s+n+m}+\|y\|^{2 s+n+m}} d^{m} y d^{n} x \\
& \geq \int_{\|x\| \leq 1} \int_{\|y\| \leq\|x\|} \frac{\sin ^{2}(\pi \xi x)}{\|x\|^{2 s+n+m}+\|x\|^{2 s+n+m}} d^{m} y d^{n} x=\frac{\omega_{m}}{2} \int_{\|x\| \leq 1} \frac{\sin ^{2}(\pi \xi x)}{\|x\|^{2 s+n}} d^{n} x,
\end{aligned}
$$

where by $\omega_{m}$ we denoted the volume of unit ball in $\mathbf{R}^{m}$. Thus we proved that

$$
\frac{\omega_{m}}{2} \int_{\|x\| \leq 1} \frac{\sin ^{2}(\pi \xi x)}{\|x\|^{2 s+n}} d^{n} x \leq A(\xi, 0)
$$

for $\xi \in \mathbf{R}^{n}$.

Now, we are in position to prove inequality (14). We have

$$
\begin{aligned}
\|u\|_{H^{s}}^{2}= & \sum_{k \in \mathbf{Z}^{m}} \int_{\mathbf{R}^{n}}\left(1+\|\xi\|^{2 s}+\|k\|^{2 s}\right)|\hat{u}(\xi, k)|^{2} d^{n} \xi \\
= & \sum_{k \neq 0} \int_{\mathbf{R}^{n}}\left(1+\|\xi\|^{2 s}+\|k\|^{2 s}\right)|\hat{u}(\xi, k)|^{2} d^{n} \xi \\
& +\int_{\mathbf{R}^{n}}\left(1+\|\xi\|^{2 s}\right)|\hat{u}(\xi, 0)|^{2} d^{n} \xi=J_{1}+J_{2} .
\end{aligned}
$$

From inequality (15) we get

$$
J_{1} \leq \max (1,1 / \widetilde{C}) \sum_{k \neq 0} \int_{\mathbf{R}^{n}}(1+A(\xi, k))|\hat{u}(\xi, k)|^{2} d^{n} \xi .
$$

Furthermore, let us recall the identity (see [31, Equality (3.12)])

$$
\int_{\mathbf{R}^{n}} \frac{\sin ^{2}(\pi \xi x)}{\|x\|^{2 s+n}} d^{n} x=C_{n, s}\|\xi\|^{2 s},
$$


where

$$
C_{n, s}=\frac{(2 \pi)^{s}}{2} \int_{\mathbf{R}^{n}} \frac{1-\cos x_{1}}{\|x\|^{n+2 s}} d x .
$$

In this way, combining the above fact with inequality (17) we have

$$
\begin{aligned}
J_{2} \leq & \|u\|_{L^{2}\left(\mathbf{R}^{n} \times \mathbf{T}^{m}\right)}+\int_{\mathbf{R}^{n}}\|\xi\|^{2 s}|\hat{u}(\xi, 0)|^{2} d^{n} \xi \\
= & \|u\|_{L^{2}\left(\mathbf{R}^{n} \times \mathbf{T}^{m}\right)}+\frac{1}{C_{n, s}} \int_{\mathbf{R}^{n}} \int_{\|x\| \leq 1} \frac{\sin ^{2}(\pi \xi x)}{\|x\|^{2 s+n}} d^{n} x|\hat{u}(\xi, 0)|^{2} d^{n} \xi \\
& +\frac{1}{C_{n, s}} \int_{\mathbf{R}^{n}} \int_{\|x\|>1} \frac{\sin ^{2}(\pi \xi x)}{\|x\|^{2 s+n}} d^{n} x|\hat{u}(\xi, 0)|^{2} d^{n} \xi \\
\leq & \|u\|_{L^{2}\left(\mathbf{R}^{n} \times \mathbf{T}^{m}\right)}+\frac{2}{C_{n, s} C_{m}} \int_{\mathbf{R}^{n}} A(\xi, 0)|\hat{u}(\xi, 0)|^{2} d^{n} \xi+\frac{C_{2}}{C_{n, s}} \int_{\mathbf{R}^{n}}|\hat{u}(\xi, 0)|^{2} d^{n} \xi,
\end{aligned}
$$

where

$$
C_{2}=\int_{\|x\|>1} \frac{1}{\|x\|^{2 s+n}} d^{n} x
$$

Hence, we obtain

$$
J_{2} \leq \max \left(\frac{2}{C_{n, s} C_{m}}, 1+\frac{C_{2}}{C_{n, s}}\right)\left(\int_{\mathbf{R}^{n}} A(\xi, 0)|\hat{u}(\xi, 0)|^{2} d^{n} \xi+\|u\|_{L^{2}\left(\mathbf{R}^{n} \times \mathbf{T}^{m}\right)}^{2}\right) .
$$

Combining estimates for $J_{1}$ and $J_{2}$ we get

$$
\begin{aligned}
\|u\|_{H^{s}}^{2} \leq & \left(\max \left(1, \frac{1}{\widetilde{C}}\right)+\max \left(\frac{2}{C_{n, s} C_{m}}, 1+\frac{C_{2}}{C_{n, s}}\right)\right) \\
& \left.\cdot\left(\sum_{k \in \mathbf{Z}^{m}} \int_{\mathbf{R}^{n}} A(\xi, k)\right)|\hat{u}(\xi, k)|^{2} d^{n} \xi+\|u\|_{L^{2}\left(\mathbf{R}^{n} \times \mathbf{T}^{m}\right)}^{2}\right) .
\end{aligned}
$$

Therefore, taking into account identity (12) from the proof of Theorem 6.1 , we get inequality (14) and this finishes the proof of the theorem.

Next, we consider a little more exotic example. Namely, we shall prove the equivalence of fractional Sobolev and Sobolev spaces on $\mathbf{Q}_{p}^{n}$.

Theorem 6.3. Let $s \in(0,1)$, then

$$
\mathcal{H}_{s, n}\left(\mathbf{Q}_{p}^{n}\right) \simeq H^{s}\left(\mathbf{Q}_{p}^{n}\right) \text {. }
$$

Proof. Since the Haar measure on $\mathbf{Q}_{p}^{n}$ is $n$-regular, from Theorem 6.1 we have $H^{s}\left(\mathbf{Q}_{p}^{n}\right) \hookrightarrow \mathcal{H}_{s, n}\left(\mathbf{Q}_{p}^{n}\right)$. Thus we need to prove the opposite embedding. But let us see that it is enough to show the existence of a constant $C=C(n, p, s)$ such that for all $\xi \in \mathbf{Q}_{p}^{n}$

$$
A(\xi) \geq C\|\xi\|_{p}^{2 s}
$$

where

$$
A(\xi)=\int_{\mathbf{Q}_{p}^{n}} \frac{|\xi(x)-1|^{2}}{\|x\|_{p}^{2 s+n}} d x, \quad \xi \in \mathbf{Q}_{p}^{n} .
$$

Let us fix $\xi \in \mathbf{Q}_{p}^{n}$. If $\xi=0$, then inequality (18) is satisfied. Thus, we assume that $\left|\xi_{1}\right|_{p}=\|\xi\|_{p}$. Note that simple algebraic operations give

$$
|\xi(x)-1|^{2}=4 \sin ^{2}\left(\pi\{\xi \cdot x\}_{p}\right),
$$


where $\{\cdot\}_{p}$ is a $p$-adic fractional part. Subsequently, we define the following set

$$
D=\left\{x \in \mathbf{Q}_{p}^{n}:\left|\xi_{1} x_{1}\right|_{p}=p\right\} \cap\left\{x \in \mathbf{Q}_{p}^{n}:\left|x_{1}\right|_{p}>\left|x_{2}\right|_{p}=\max _{2 \leq i \leq n}\left|x_{i}\right|_{p}\right\} .
$$

For $x \in D$ we have

$$
\left|\xi_{2} x_{2}+\cdots+\xi_{n} x_{n}\right|_{p} \leq \max _{2 \leq i \leq n}\left|\xi_{i} x_{i}\right|_{p} \leq\left|\xi_{1}\right|_{p}\left|x_{2}\right|_{p}<\left|\xi_{1}\right|_{p}\left|x_{1}\right|_{p}=p .
$$

Thus

$$
|\xi \cdot x|_{p}=\left|\xi_{1} x_{1}\right|_{p}=p
$$

Hence, for $x \in D$ we have

$$
p^{-1} \leq\{\xi \cdot x\}_{p} \leq 1-p^{-1}
$$

and

It follows that

$$
\sin ^{2}\left(\pi\{\xi \cdot x\}_{p}\right) \geq \sin ^{2}\left(\pi p^{-1}\right)=\sin ^{2}\left(\pi\left|\xi_{1} x_{1}\right|_{p}^{-1}\right) .
$$

$$
A(\xi) \geq \int_{D} \frac{4 \sin ^{2}\left(\pi\left|\xi_{1} x_{1}\right|_{p}^{-1}\right)}{\|x\|_{p}^{2 s+n}} d^{n} x .
$$

Next, exploring the change of variables formula with $z_{1}=\xi_{1} x_{1}, z_{2}=\xi_{1} x_{2}, \ldots, z_{n}=$ $\xi_{1} x_{n}$ and jacobian $\left|\xi_{1}\right|_{p}^{-n}$ we get

$$
\begin{aligned}
\int_{D} \frac{4 \sin ^{2}\left(\pi\left|\xi_{1} x_{1}\right|_{p}^{-1}\right)}{\|x\|_{p}^{2 s+n}} d^{n} x & =\int_{D^{\prime}} \frac{1}{\left|\xi_{1}\right|_{p}^{n}} \cdot \frac{4 \sin ^{2}\left(\pi\left|z_{1}\right|_{p}^{-1}\right)}{\left\|\frac{z}{\xi_{1}}\right\|_{p}^{2 s+n}} d^{n} z \\
& =\int_{D^{\prime}} \frac{\left|\xi_{1}\right|_{p}^{2 s+n}}{\left|\xi_{1}\right|_{p}^{n}} \cdot \frac{4 \sin ^{2}\left(\pi\left|z_{1}\right|_{p}^{-1}\right)}{\|z\|_{p}^{2 s+n}} d^{n} z \\
& =\|\xi\|_{p}^{2 s} \int_{D^{\prime}} \frac{4 \sin ^{2}\left(\pi\left|z_{1}\right|_{p}^{-1}\right)}{\|z\|_{p}^{2 s+n}} d^{n} z
\end{aligned}
$$

where

$$
D^{\prime}=\left\{z \in \mathbf{Q}_{p}^{n}:\left|z_{1}\right|_{p}=p\right\} \cap\left\{z \in \mathbf{Q}_{p}^{n}:\left|z_{1}\right|_{p}>\left|z_{2}\right|_{p}=\max _{2 \leq i \leq n}\left|z_{i}\right|_{p}\right\} .
$$

Furthermore, by the elementary inequalities

$$
\left|z_{1}\right|_{p}^{-1}=p^{-1} \leq \frac{1}{2}, \quad \sin x \geq \frac{2}{\pi} x \quad \text { for } x \in\left(0, \frac{\pi}{2}\right)
$$

we get

$$
\int_{D^{\prime}} \frac{4 \sin ^{2}\left(\pi\left|z_{1}\right|_{p}^{-1}\right)}{\|z\|_{p}^{2 s+n}} d^{n} z \geq 16 \int_{D^{\prime}} \frac{\left|z_{1}\right|_{p}^{-2}}{\|z\|_{p}^{2 s+n}} d^{n} z=16 I .
$$

Denoting

$$
S_{k}^{1}=\left\{z \in \mathbf{Q}_{p}:|z|_{p}=p^{k}\right\}
$$

we get

$$
S_{1}^{1} \times S_{0}^{1} \times \cdots \times S_{0}^{1} \subset D^{\prime}
$$

Since $\left|z_{1}\right|_{p}=\|z\|_{p}$, by the Fubini theorem we get

$$
\begin{aligned}
I & =\int_{D^{\prime}} \frac{d^{n} z}{\|z\|_{p}^{2 s+n+2}}=\frac{1}{p^{2 s+n+2}} \int_{D^{\prime}} d^{n} z \\
& \geq \frac{1}{p^{2 s+n+2}} \int_{S_{1}^{1} \times S_{0}^{1} \times \cdots \times S_{0}^{1}} d^{n} z=\frac{1}{p^{2 s+n+2}} \int_{S_{1}^{1}} d z_{1}\left(\int_{S_{0}^{1}} d z\right)^{n-1} .
\end{aligned}
$$


Finally, the fact that $\mu\left(S_{k}^{1}\right)=p^{k}-p^{k-1}$ leads us to the inequality

$$
I \geq \frac{1}{p^{2 s+n+2}}(p-1)\left(p^{0}-p^{-1}\right)^{n-1}=\frac{(p-1)^{n}}{p^{2 s+2 n+1}} .
$$

This finishes the proof of (18) and the theorem follows.

\section{Appendix}

Let $\mathbf{Q}$ be the set of all rational numbers and let $p \in \mathbf{N}$ be any prime number. We define $p$-adic additive valuation $v_{p}$ in the following way:

(i) If $x \in \mathbf{Z}$, then $v_{p}(x)$ is equal to the highest power of $p$ which divides $x$.

(ii) If $x=a / b$, where $a, b \in \mathbf{Z}$, then $v_{p}(x)=v_{p}(a)-v_{p}(b)$.

(iii) We set $v_{p}(0)=+\infty$.

Next we define a map $|\cdot|_{p}: \mathbf{Q} \rightarrow \mathbf{R}_{+}$

$$
|x|_{p}:= \begin{cases}p^{-v_{p}(x)}, & \text { if } x \neq 0 \\ 0, & \text { if } x=0\end{cases}
$$

This map is a non-Archimedean norm ${ }^{8}$ on $\mathbf{Q}$. We define the field of $p$-adic numbers $\mathbf{Q}_{p}$ as the completion of the field of rational numbers $\mathbf{Q}$ in $p$-adic norm (for details of this construction see $[3,34])$. The $p$-adic norm $|\cdot|_{p}$ extends to the norm on the whole $\mathbf{Q}_{p}$. The set of values which this norm takes is discrete and equal to

$$
\left\{p^{n}: n \in \mathbf{Z}\right\} \cup\{0\} .
$$

The set $\mathbf{Q}_{p}$ together with $p$-adic addition and topology induced by $p$-adic norm is a locally compact abelian group. We choose a unique Haar measure on $\mathbf{Q}_{p}$ satisfying the condition

$$
\int_{\mathbf{Z}_{p}} d x=1
$$

where $\mathbf{Z}_{p}:=B_{1}=\left\{x \in \mathbf{Q}_{p}:|x|_{p} \leq 1\right\}$ is the set of $p$-adic integers. Denoting by $\mu$ the Haar measure on $\mathbf{Q}_{p}$ we have

$$
\mu\left(B_{n}\right)=p^{n},
$$

where $B_{n}=\left\{x \in \mathbf{Q}_{p}:|x|_{p} \leq p^{n}\right\}$. Measures of spheres $S_{n}=\left\{x \in \mathbf{Q}_{p}:|x|_{p}=p^{n}\right\}$ are given by the formula

$$
\mu\left(S_{n}\right)=p^{n}(1-1 / p) .
$$

The dual group of $\mathbf{Q}_{p}$ is $\mathbf{Q}_{p}$ and each character $\xi \in \mathbf{Q}_{p}$ has the following form

$$
\xi(x)=\exp \left(2 \pi i\{\xi x\}_{p}\right),
$$

where $\{\cdot\}_{p}$ is $p$-adic fractional part i.e. each $p$-adic number $x \in \mathbf{Q}_{p}$ can be written in the following form (see [3])

$$
x=\sum_{n=v_{p}(x)}^{\infty} c_{n} p^{n},
$$

\footnotetext{
${ }^{8}$ Let $F$ be a field. A norm on $F$ is a map $\|\cdot\|: F \rightarrow \mathbf{R}_{+}$such that for all $x, y \in F$ we have (1) $\|x\|=0 \Leftrightarrow x=0 ;(2)\|x y\|=\|x\|\|y\| ;(3)\|x+y\| \leq\|x\|+\|y\|$. A norm is called non-Archimedean if it satisfies the additional condition $\left(3^{\prime}\right)\|x+y\| \leq \max \{\|x\|,\|y\|\}$.
} 
with $c_{v_{p}(x)} \neq 0$ and $c_{n} \in\{0,1,2, \ldots, p-1\}$. If $x$ has the form (19), then its $p$-adic fractional part is

$$
\{x\}_{p}= \begin{cases}\sum_{n=v_{p}(x)}^{-1} c_{n} p^{n}, & \text { if } v_{p}(x)<0 \\ 0, & \text { if } v_{p}(x) \geq 0 .\end{cases}
$$

One can easily check that if $v_{p}(x)<0$, then

$$
p^{v_{p}(x)} \leq\{x\}_{p} \leq 1-p^{v_{p}(x)} .
$$

We define $\mathbf{Q}_{p}^{n}$ as a product of $n$ copies of $\mathbf{Q}_{p}$ with the product topology and component-wise operations. Norm on this group is given by

$$
\|x\|_{p}=\max _{i=1,2, \ldots, n}\left|x_{i}\right|_{p} .
$$

The Haar measure on $\mathbf{Q}_{p}^{n}$ is the product of Haar measures on $\mathbf{Q}_{p}$. Balls $B_{k}^{n}(a)=$ $\left\{x \in \mathbf{Q}_{p}^{n}:\|x-a\|_{p} \leq p^{k}\right\}$ are product of balls $B_{k}\left(a_{i}\right)$ in $\mathbf{Q}_{p}$ i.e.

$$
B_{k}^{n}(a)=B_{k}\left(a_{1}\right) \times \cdots \times B_{k}\left(a_{n}\right),
$$

where $B_{k}\left(a_{i}\right)=\left\{x \in \mathbf{Q}_{p}:\left|x-a_{i}\right|_{p} \leq p^{k}\right\}$.

For $x, y \in \mathbf{Q}_{p}^{n}$ we define $x \cdot y=x_{1} y_{1}+\cdots+x_{n} y_{n}$, and every character on $\mathbf{Q}_{p}^{n}$ is of the form

$$
\xi(x)=\exp \left(i 2 \pi\{\xi \cdot x\}_{p}\right)
$$

Finally, we recall the change of variables formula.

Theorem 7.1. [3] If $x$ is an analytic diffeomorphism of a clopen set $K_{1} \subset \mathbf{Q}_{p}^{n}$ onto $K \subset \mathbf{Q}_{p}^{n}$, and

$$
\operatorname{det}\left(\frac{\partial x(y)}{\partial y}\right)=\operatorname{det}\left(\frac{\partial x_{k}(y)}{\partial y_{j}}\right) \neq 0, \quad y \in K_{1},
$$

then for any $f \in L^{1}(K)$ we have

$$
\int_{K} f(x) d^{n} x=\int_{K_{1}} f(x(y))\left|\operatorname{det}\left(\frac{\partial x(y)}{\partial y}\right)\right|_{p} d^{n} y .
$$

Acknowledgements. P. G. was partially supported by a grant Iuventus Plus of the Ministry of Science and Higher Education of the Republic of Poland, Nr 0009/IP3/ 2015/73. Some part of this research has been performed during the staying of T. K. in Residencia Erasmo of the Universidad Autónoma de Madrid. T. K. thanks the Organisers of the Research Term on Analysis and Geometry in Metric Spaces for their hospitality. Special thanks goes to the pde cyclist group. Moreover, the authors wishs to thank the referees for comments and suggestions.

\section{References}

[1] Adamowicz, T., M. Gaczkowski, and P. Górka: Harmonic functions on metric measure spaces. - Submitted.

[2] Adams, R. A.: Sobolev spaces. - Academic Press, New York, 1975.

[3] Albeverio, S., A. Yu. Khrennikov, and V. Shelkovich: Theory of $p$-adic distributions: linear and nonlinear models. - London Math. Soc. Lecture Note Ser. 370, Cambridge Univ. Press, Cambridge, 2010.

[4] Aronszajn, N.: Boundary values of functions with finite Dirichlet integral. - Tech. Report 14, Univ. of Kansas 1955, 77-94.

[5] Aubin, T.: Some nonlinear problems in Riemannian geometry. - Springer-Verlag, Berlin, 1998. 
[6] Bahouri, H., C. Fermanian-Kammerer, and I. Gallagher: Analyse de l'espace des phases et calcul pseudo-differential sur le groupe de Heisenberg. - C. R. Math. Acad. Sci. Paris 347:17-18, 2009, 1021-1024.

[7] BÉnyi, Á., and T. Oн: The Sobolev inequality on the torus revisited. - Publ. Math. Debrecen $83: 3,2013,359-374$.

[8] Bourbaki, N.: Théories spectrales: Chapitres 1 et 2. - Hermann, 1967.

[9] Bruhat, F.: Distributions sur un groupe localement compact et applications à l'étude des répresentations des groupes p-adiques. - Bulletin de la S.M.F. 89, 1961, 43-75.

[10] Capogna, L., D. Danielli, S. D. Pauls, and J. Tyson: An introduction to the Heisenberg group and the sub-Riemannian isoperimetric problem. - Progr. Math. 259, Birkhäuser Verlag, Basel, 2007.

[11] Deitmar, A., and S. Echterhoff: Principles of harmonic analysis. - Springer, 2009.

[12] Gaczkowski, M., and P. Górka: Harmonic functions on metric measure spaces: convergence and compactness. - Potential Anal. 31, 2009, 203-214.

[13] Gagliardo, E.: Caratterizzazioni delle tracce sulla frontiera relative ad alcune classi di funzioni in $n$ variabili. - Rend. Sem. Mat. Univ. Padova 27, 1957, 284-305.

[14] Gagliardo, E.: Proprietà di alcune classi di funzioni in più variabili. - Ricerche Mat. 7, 1958, $102-137$.

[15] GórkA, P.: Pego theorem on locally compact abelian groups. - J. Algebra Appl. 13, 2014, 1350143.

[16] Górka, P., and T. Kostrzewa: Pego everywhere. - J. Algebra Appl. 15, 2016, 1650074.

[17] Górka, P., and T. Kostrzewa: Sobolev spaces on metrizable groups. - Ann. Acad. Sci. Fenn. Math. 40, 2015, 837-849.

[18] Górka, P., T. Kostrzewa, and E. G. Reyes: The Rellich lemma on compact abelian groups and equations of infinite order. - Int. J. Geom. Meth. Mod. Phys. 10, 2013, 1220030, 1-11.

[19] Górka, P., T. Kostrzewa, and E. G. Reyes: Sobolev spaces on locally compact Abelian groups: compact embeddings and local spaces. - J. Funct. Spaces 2014, 2014, 404738.

[20] Górka, P., and E. G. Reyes: Sobolev spaces on locally compact abelian groups and the bosonic string equation. - J. Aust. Math. Soc. 98, 2015, 39-53.

[21] HajŁAsz, P.: Sobolev spaces on an arbitrary metric space. - Potential Anal. 5, 1996, 403-415.

[22] HajŁasz, P., and P. Koskela: Sobolev met Poincaré. - Mem. Amer. Math. Soc. 145:688, 2000.

[23] Hebey, E.: - Sobolev spaces on Riemannian manifolds. - Lecture Notes in Math. 1635, Springer-Verlag, Berlin, 1996.

[24] Hewitt, E., and K. A. Ross: Abstract harmonic analysis: Volumes I and II. - Grundlehren Math. Wiss., Springer-Verlag, New York-Berlin, 1963 and 1970.

[25] Montgomery, D., and L. Zippin: Topological transformation groups. - Interscience Publishers, New York-London, 1955.

[26] Heinonen, J.: Lectures on analysis on metric spaces. - Universitext, Springer-Verlag, New York, 2001.

[27] Heyer, H.: Probability measures on locally compact groups. - Springer-Verlag, BerlinHeidelberg, 1977.

[28] KIM, Y.: A simple proof of the $p$-adic version of the Sobolev embedding theorem. Commun. Korean Math. Soc. 25, 2010, 27-36.

[29] Kostrzewa, T.: Przestrzenie Sobolewa na grupach metrycznych. - PhD Thesis, Warsaw University of Technology, 2019 (in Polish).

[30] Meyers, N. G., and J. Serrin: H = W. - Proc. Nat. Acad. Sci. U.S.A. 51, 1964, 1055-1056. 
[31] Di Nezza, E., G. Palatucci, and E. Valdinoci: Hitchhiker's guide to the fractional Sobolev spaces. - Bull. Sci. Math. 136, 2012, 521-573.

[32] Osborne, M.S.: On the Schwartz-Bruhat space and the Paley-Wiener theorem for locally compact Abelian groups. - J. Funct. Anal. 19, 1975, 40-49.

[33] Rodriguez-Vega, J. J., and W. A. Zuniga-Galindo: Elliptic pseudo-differential equations and Sobolev spaces over p-adic fields. - Pacific J. Math. 246, 2010, 407-420.

[34] Robert, A. M.: - A course in p-adic analysis. - Grad. Texts in Math. 198, Springer-Verlag, New York, 2000.

[35] Ruzhansky, M., and V. Turunen: Pseudo-differential operators and symmetries. Background analysis and advanced topics. - Pseudo Diff. Oper. 2, Birkhäuser Verlag, Basel, 2010.

[36] Shanmugalingam, N.: Newtonian spaces: an extension of Sobolev spaces to metric measure spaces. - Rev. Mat. Iberoamericana 16, 2000, 243-279.

[37] Shanmugalingam, N.: Harmonic functions on metric spaces. - Illinois J. Math. 45, 2001, $1021-1050$.

[38] SLobodeckiř, L. N.: Generalized Sobolev spaces and their application to boundary problems for partial differential equations. - Leningrad. Gos. Ped. Inst. Učen. Zap. 197, 1958, 54-112.

[39] Struble, R. A.: Metrics in locally compact groups. - Compositio Math. 28, 1974, 217-222.

[40] Taibleson, M. H.: Fourier analysis on local fields. - Mathematical Notes 15, Princeton Univ. Press and Univ. of Tokyo Press, Princeton, NJ, 1975.

[41] TARTAR, L.: An introduction to Sobolev spaces and interpolation spaces. - Lect. Notes Unione Mat. Ital. 3, Springer-Verlag, Berlin Heidelberg, 2007.

[42] Taylor, M. E.: Partial differential equations: Volume I. - Springer-Verlag, 1996.

Received 28 September 2018 • Accepted 27 February 2019 